\title{
Limits on the presence of planets in systems with debris discs: HD 92945 and HD 107146
}

D. Mesa, ${ }^{1 \star}$ S. Marino ${ }^{\circledR},{ }^{2,3}$ M. Bonavita ${ }^{\circledR}, 1,4,5$ C. Lazzoni, ${ }^{1,6}$ C. Fontanive ${ }^{\circledR},{ }^{1,4,7}$ S. Pérez, ${ }^{8}$ V. D’ Orazi, ${ }^{1,9}$ S. Desidera, ${ }^{1}$ R. Gratton, ${ }^{1}$ N. Engler, ${ }^{10}$ T. Henning, ${ }^{2}$ M. Janson, ${ }^{2,11}$ Q. Kral, ${ }^{12}$ M. Langlois, ${ }^{13,14}$ S. Messina, ${ }^{15}$ J. Milli, ${ }^{16}$ N. Pawellek ${ }^{\oplus},{ }^{3,17}$ C. Perrot, ${ }^{12,18,19}$ E. Rigliaco, ${ }^{1}$ E. Rickman, ${ }^{20,21}$ V. Squicciarini, ${ }^{1,6}$ A. Vigan, ${ }^{14}$ Z. Wahhaj, ${ }^{14,22}$ A. Zurlo, ${ }^{14,23,24}$ A. Boccaletti, ${ }^{12}$ M. Bonnefoy, ${ }^{16}$ G. Chauvin, ${ }^{16,25}$ V. De Caprio, ${ }^{26}$ M. Feldt, ${ }^{2}$ L. Gluck, ${ }^{16}$ J. Hagelberg ${ }^{\circledR},{ }^{20}$ M. Keppler, ${ }^{2}$ A.-M. Lagrange, ${ }^{16}$ R. Launhardt, ${ }^{2}$ A.-L. Maire, ${ }^{27}$ M. Meyer, ${ }^{10,28}$ O. Moeller-Nilsson, ${ }^{2}$ A. Pavlov, ${ }^{2}$ M. Samland, ${ }^{2,11}$ T. Schmidt ${ }^{12}$ and L. Weber ${ }^{20}$

Affiliations are listed at the end of the paper

Accepted 2021 February 5. Received 2021 February 3; in original form 2020 October 16

\begin{abstract}
Recent observations of resolved cold debris discs at tens of au have revealed that gaps could be a common feature in these Kuiper-belt analogues. Such gaps could be evidence for the presence of planets within the gaps or closer in near the edges of the disc. We present SPHERE observations of HD 92945 and HD 107146, two systems with detected gaps. We constrained the mass of possible companions responsible for the gap to 1-2 $M_{\text {Jup }}$ for planets located inside the gap and to less than $5 M_{\text {Jup }}$ for separations down to 20 au from the host star. These limits allow us to exclude some of the possible configurations of the planetary systems proposed to explain the shape of the discs around these two stars. In order to put tighter limits on the mass at very short separations from the star, where direct-imaging data are less effective, we also combined our data with astrometric measurements from Hipparcos and Gaia and radial-velocity measurements. We were able to limit the separation and the mass of the companion potentially responsible for the proper-motion anomaly of HD 107146 to values of 2-7 au and 2-5 $M_{\text {Jup }}$, respectively.
\end{abstract}

Key words: instrumentation: spectrographs - methods: data analysis - techniques: imaging spectroscopy - planetary systems.

\section{INTRODUCTION}

Protoplanetary discs around young (a few Myr) stars are nowadays considered formation environments for planetary systems (Chen et al. 2012; Marshall et al. 2014). In recent years, a growing number of structures, e.g. rings, gaps, and asymmetries, have been identified in these discs (e.g. ALMA Partnership et al. 2015; van Boekel et al. 2017; Fedele et al. 2018; Isella et al. 2018; Pinilla et al. 2018). While different mechanisms have been proposed to explain these structures, one of the most promising is the one implying the presence of planetary mass objects still in their formation process. However, at the moment, the detection of planets associated with these structures has been confirmed for just a handful of cases. The most striking of these detections is certainly the PDS 70 system, in which two planetary companions have been detected through direct imaging in the gap of the disc (Keppler et al. 2018; Müller et al. 2018; Haffert et al. 2019; Mesa et al. 2019a).

At older ages (from tens to hundreds Myr), debris discs are expected to have lost a large part of their gaseous component, while their dust component is continuously replenished by collisions of

^E-mail: dino.mesa@inaf.it small bodies orbiting within Kuiper-belt analogues. The presence of planets can shape these discs as demonstrated by well known cases like $\beta$ Pic (Lagrange et al. 2009), HR 8799 (Marois et al. 2008, 2010; Booth et al. 2016; Read et al. 2018), and HD 95086 (Rameau et al. 2013, 2016; Su et al. 2015). This is why asymmetries detected in the debris disc are often considered hints to the presence of planetary companions (see e.g. Kalas, Graham \& Clampin 2005; Dent et al. 2014; Boccaletti et al. 2015). Similarly, the existence of wide-cavity interiors to cold debris belts or in between warm asteroidbelt analogues and cold outer belts has been used to argue for the presence of multiple planets clearing those wide regions of debris, in analogy to the Solar system architecture (Apai et al. 2008; Meshkat et al. 2015; Shannon et al. 2016; Lazzoni et al. 2018; Matthews et al. 2018; Lombart et al. 2020). Perhaps the most convincing and constraining evidence for planets at tens of au is the presence of gaps in broad cold debris discs. There is a growing number of gapped debris discs, e.g. HD 107146 (Ricci et al. 2015; Marino et al. 2018), HD 92945 (Golimowski et al. 2011; Marino et al. 2019), HD 15115 (MacGregor et al. 2019; Engler et al. 2019), and HD 206893 (Marino et al. 2020, Nederlander et al., submitted) seen with ALMA tracing the distribution of mm-sized grains. Scattered light images have also revealed the presence of gaps in a few systems, e.g. HD 131835 (Feldt et al. 2017), HD 141569 (Perrot et al. 2016), HD 120326 
(Bonnefoy et al. 2017), and NZLup (Boccaletti et al. 2019), but these observations trace small $\mu \mathrm{m}$-sized dust particles, which are not always a good tracer for the true mass distribution in the disc (e.g. Wyatt 2006).

One of the simplest interpretations for these gaps is that they are caused by single planets orbiting in them. It is well known that a planet embedded in a debris disc will carve a gap around its orbit, where particles become unstable and are scattered away by the planet. This is the so-called chaotic zone (Wisdom 1980) and its size has been studied extensively both exterior to a planet's orbit (Quillen \& Faber 2006; Nesvold \& Kuchner 2015) and interior to it (Morrison \& Malhotra 2015). Roughly speaking, we expect that the gap size will have a radial width proportional to $\mu^{2 / 7}$, where $\mu$ is the mass ratio between the planet and the star. Therefore the width of a given gap can be directly linked to the mass of the putative planet carving it.

However, gaps can also be carved by planets interior to the disc inner edges. As shown by Pearce \& Wyatt (2015) the secular interaction and scattering between a planet on an eccentric orbit and a disc with a similar mass further out can open a wide asymmetric gap in the disc. Another possibility is that two or multiple planets closer in could place a secular resonance within the disc span, creating a gap in the disc as well (Marino et al. 2018). Moreover, as shown more recently by Sefilian, Rafikov \& Wyatt (2020), if we consider the non-negligible mass of debris discs and their self-gravity, the secular interaction between the disc and a single eccentric planet closer in can also place a secular resonance within the disc, opening an asymmetric gap (see also Zheng et al. 2017). Although HD 107146's gap is very symmetric and thus unlikely to be carved by the alternative scenarios described above (Marino et al. 2018), these could potentially explain the gaps around HD 92945, HD 15115, and HD 206893. In fact HD 206893 is known to host a brown dwarf orbiting at 11 au, and Sefilian et al. (2020) showed that this could explain the gap location given the estimated disc mass for that system. Another possible scenario to explain gaps in the distribution of $\mu \mathrm{m}$-sized grains involves planet migration and resonant trapping as proposed by Wyatt (2006). This scenario was used by Golimowski et al. (2011) to explain the structure of the HD 92945 debris ring. However, this scenario only works for $\mu \mathrm{m}$-sized grains seen in scattered light and the gap seen also at $\mathrm{mm}$ wavelengths rules it out as a possible mechanism.

Tight upper limits on the masses of possible companions, both inside and outside the gap in the disc, could then allow these possible scenarios to be constrained. This would help the understanding of the evolutive processes behind the formation of these structures. While present high-contrast imagers like SPHERE (Beuzit et al. 2019) or GPI (Macintosh et al. 2006) are only able to detect planets more massive than $1 M_{\text {Jup }}$, their data can still place meaningful upper limits on the masses of the companions carving the gaps. In this work we present SPHERE observations of two stars hosting debris discs with gaps, HD 92945 and HD 107146, and we use them to set mass limits on companions within a few tens of au from the stars.

Furthermore, to complement at shorter separations the limits obtained through direct imaging, we have exploited measurements of the proper-motion anomaly (PMa) obtained by Kervella et al. (2019) and radial-velocity (RV) data for these stars.

The paper presents a description of the two targets in Section 2 and describes the observations and data reduction in Section 3. In Section 4 we describe the results of our analysis while in Section 5 we discuss the results; in Section 6 we give our conclusion.

\section{TARGET PROPERTIES}

\subsection{HD 92945}

HD 92945 is a K1V (Torres et al. 2006) star at a distance from the Sun of $21.54 \pm 0.02$ pc (Gaia Collaboration 2016, 2018).

\subsubsection{Spectroscopic analysis}

In order to constrain the stellar properties, we analysed a FEROS (Kaufer et al. 1999) spectrum from the ESO archive taken on 2018 April 21 under the open-time programme 0101.A-9012(A) (PI $\mathrm{R}$. Launhardt). The spectrum covers a wavelength range between $\approx 3600$ and $9000 \AA$ with a nominal resolution of $R=48000$; the median signal-to-noise ratio (SNR) per pixel is 260. We carried out continuum normalization and rest-frame correction using the 2020 version of ISPEC (Blanco-Cuaresma et al. 2014, 2019); equivalent widths (EWs) have been measured with ARESv2 (Sousa et al. 2015). Atmospheric parameters and metallicity ([Fe/H]) have been obtained through EW measurements of iron lines with the $q^{2}$ PYTHON wrapper of MOOG (Sneden 1973, 2017 version), developed and maintened by I. Ramírez (Ramírez et al. 2014). We used the ODFNEW ATLAS9 set of model atmopsheres by Castelli \& Kurucz (2003) and the line list published in D'Orazi et al. (2020). Our analysis results in the following parameters: $T_{\text {eff }}=5147 \pm 40 \mathrm{~K}$, $\log g=4.46 \pm 0.13$ dex, microturbulent velocity $1.15 \pm 0.12 \mathrm{~km} \mathrm{~s}^{-1}$, and $[\mathrm{Fe} / \mathrm{H}]=-0.01 \pm 0.03$ dex. Broad-band colours $(B-V, V-I$, $B_{\mathrm{p}}-R_{\mathrm{p}}$, and $\left.V-K\right)$ are consistent with the spectroscopic estimate of about $\approx 5200 \mathrm{~K}$ from the tables in Pecaut \& Mamajek (2013).

\subsubsection{Stellar age}

There are various recent estimates of the age of the star, typically between 100 and 300 Myr (e.g. Song, Zuckerman \& Bessell 2004; Plavchan et al. 2009; Vigan et al. 2017). Nielsen et al. (2019) quote membership of AB Dor with an estimated age of $149_{-49}^{+31}$ Myr (Bell, Mamajek \& Naylor 2015; Vigan et al. 2017). We reconsidered the available age indicators.

We searched for the stellar rotation period by exploiting the photometric variability in the TESS data (Ricker et al. 2015). We considered the TESS pre-search data conditioning simple aperture photometry (PDCSAP) time series, removing data in the time interval 1555-1557 (BJD-2457000), where residual instrumental effects were significant. The period search was carried out by computing the Lomb-Scargle periodogram (Scargle 1982; Press et al. 2002) and the CLEAN (Roberts, Lehar \& Dreher 1987) periodogram, which has the capability of effectively removing possible beat frequencies arising from the data sampling. As shown in the bottom panels of Fig. 1, the Lomb-Scargle (LS) periodogram revealed a major power peak at $P=$ $3.40 \pm 0.24 \mathrm{~d}$ (while the CLEAN procedure shows $P=3.39 \pm 0.24 \mathrm{~d}$ ), with a false alarm probability (FAP) of the order of $10^{-6}$. However, a visual inspection of the top panel of Fig. 1 shows the light curve to be double dip, owing to spots on opposite hemispheres. Therefore, the rotation period is likely $P=6.8 \pm 0.34 \mathrm{~d}$. This value is in agreement with the $P=7.176 \mathrm{~d}$ derived using the period-activity relation from Noyes et al. (1984). We mark with red and black downward arrows the epochs of the primary $(\mathrm{P})$ and secondary $(\mathrm{S})$ minima computed according to a rotation period $P=6.8 \mathrm{~d}$. We note a significant evolution of the secondary spot, which produces progressively deeper minima from one rotation cycle to the next, as well as showing a slight migration towards earlier epochs, as shown by the position of the vertical 

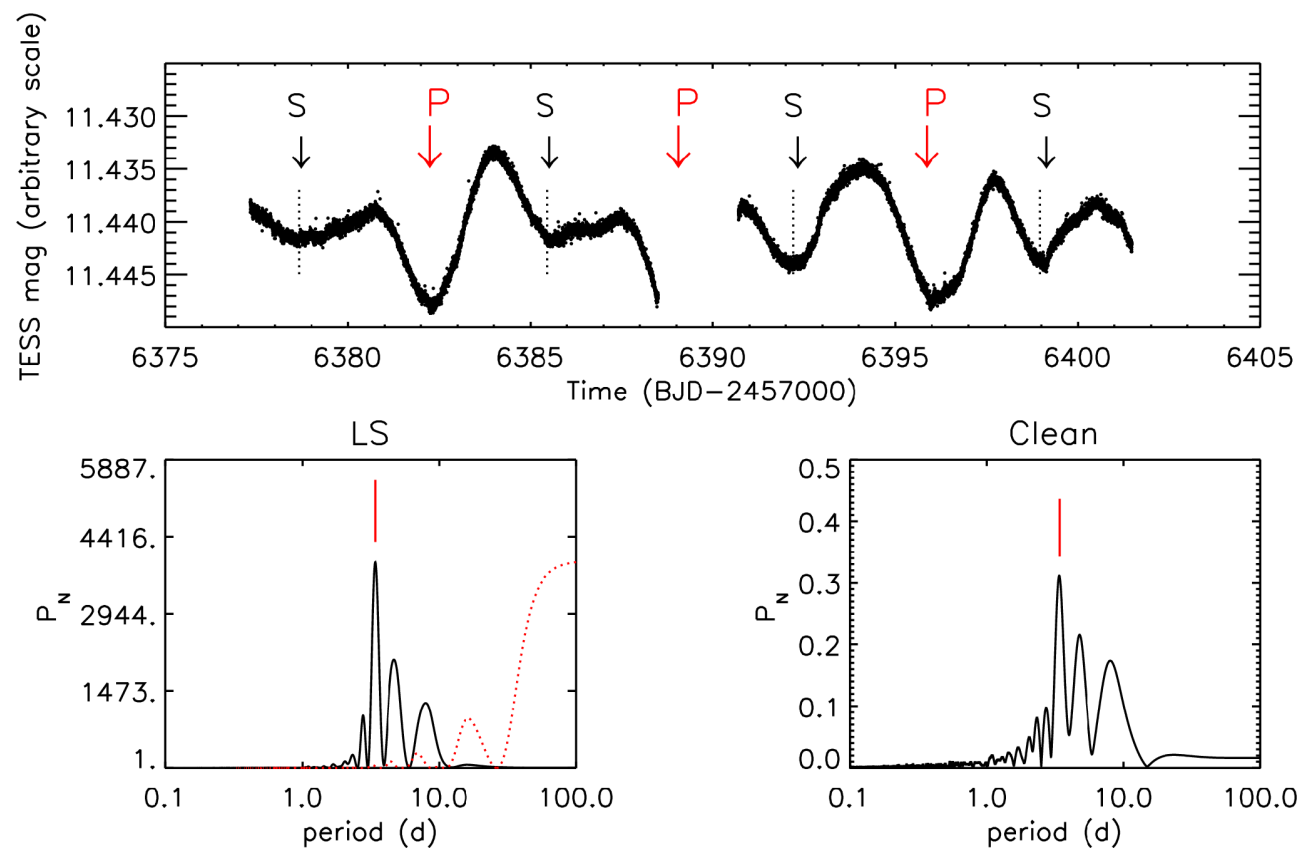

Figure 1. Results of periodogram analysis of HD 92945 with TESS data. Top panel: magnitudes versus barycentric Julian date. Red and black downward arrows mark the position of primary $(\mathrm{P})$ and secondary $(\mathrm{S})$ spots, respectively, in a double-dip light curve. Vertical dotted lines mark the epochs of observed secondary minima, which are receding with respect to the computed epochs of minima (black arrows). Bottom panels: Lomb-Scargle (left) and CLEAN (right) normalized periodograms $\left(P_{\mathrm{N}}\right)$. The red dotted line shows the spectral window function superimposed on the LS periodogram and the solid vertical lines mark the power peaks at half rotation period.

dotted lines with respect to the black downward arrows. A photometric period that is roughly two times our value was published by Strassmeier et al. (2000). We also note that our rotation period is fully compatible with the other age indicators and with the observed projected rotational velocity, at odds with a $1 / 2 \times$ or $2 \times$ period. The FAP associated with our detected period, which is the probability that a peak of given height in the periodogram is caused simply by statistical variations, i.e. Gaussian noise, was computed through Monte Carlo simulations, i.e. by generating 1000 artificial light curves obtained from the real light curve, keeping the date but permuting the magnitude values (see e.g. Herbst et al. 2002). We followed the method used by Lamm et al. (2004) to compute the errors associated with the period determination.

We found that the rotation period falls close to but slightly above the sequence of the Pleiades (Rebull et al. 2016) and between the rotation period distributions of similar colour stars of the M35 and M34 open clusters (ages 150 and 220 Myr, respectively; Meibom, Mathieu \& Stassun 2009; Meibom et al. 2011).

The chromospheric emission $\left(\log R_{\mathrm{HK}}^{\prime}=-4.32\right.$; Wright et al. $2004)$ is within the distribution of the Pleiades, while the $X$ ray emission $\left(\log L_{X} / L_{\mathrm{bol}}=-4.47\right)$ is below the Pleiades locus. The corresponding ages using Mamajek \& Hillenbrand's (2008) calibrations are 160 and $310 \mathrm{Myr}$, respectively.

The equivalent width of the $\mathrm{Li} 6708 \AA$ doublet measured on the FEROS spectrum is $153.2 \pm 1.2 \mathrm{~m} \AA$, within the observed distribution of Pleiades members but slightly below the mean values for the cluster.

Kinematic analysis using the BANYAN ${ }^{1} \Sigma$ online tool (Gagné et al. 2018) yields a null membership probability for AB Dor and other young moving groups.
All of these findings are compatible with an object with an age close to but slightly older than the Pleiades. From a weighted combination of the available indicators (Desidera et al. 2015), we adopt 170 Myr with age limits 120-250 Myr.

\subsubsection{Mass, radius, and inclination}

The stellar mass derived using the PARAM ${ }^{2}$ interface (da Silva et al. 2006), adopting the spectroscopic effective temperature and metallicity and considering only the age range for the indirect methods as in Desidera et al. (2015), is $0.86 \pm 0.01 \mathrm{M}_{\odot}$. A stellar radius of $0.75 \pm 0.02 R_{\odot}$ is also derived in the fit. Coupling this with the observed rotation period and the projected rotational velocity $v \sin i=4.5 \pm 1.0 \mathrm{~km} \mathrm{~s}^{-1}$ (Nordström et al. 2004; Valenti \& Fischer 2005), we infer an inclination of $53.7_{-12.4}^{+31.3} \mathrm{deg}$. This is compatible with the inclination of the disc (see below), although the uncertainties also allow a significant misalignment.

\subsubsection{The debris disc}

The presence of a debris disc around this star was first inferred by Silverstone (2000) based on the excess at $60 \mu \mathrm{m}$ measured by IRAS. Mid-infrared and submillimetre data obtained using Spitzer and the Caltech Submillimeter Observatory also revealed excesses at $70 \mu \mathrm{m}$ and $350 \mu \mathrm{m}$ consistent with an optically thin disc with an inner radius of 15-23 au and a temperature of 40-46 K (Chen et al. 2005; Plavchan et al. 2009). The disc was resolved for the first time by Golimowski et al. (2011) using Hubble Space Telescope (HST) images in the $V$ and $I$ bands. They also estimated the fractional infrared luminosity of the 

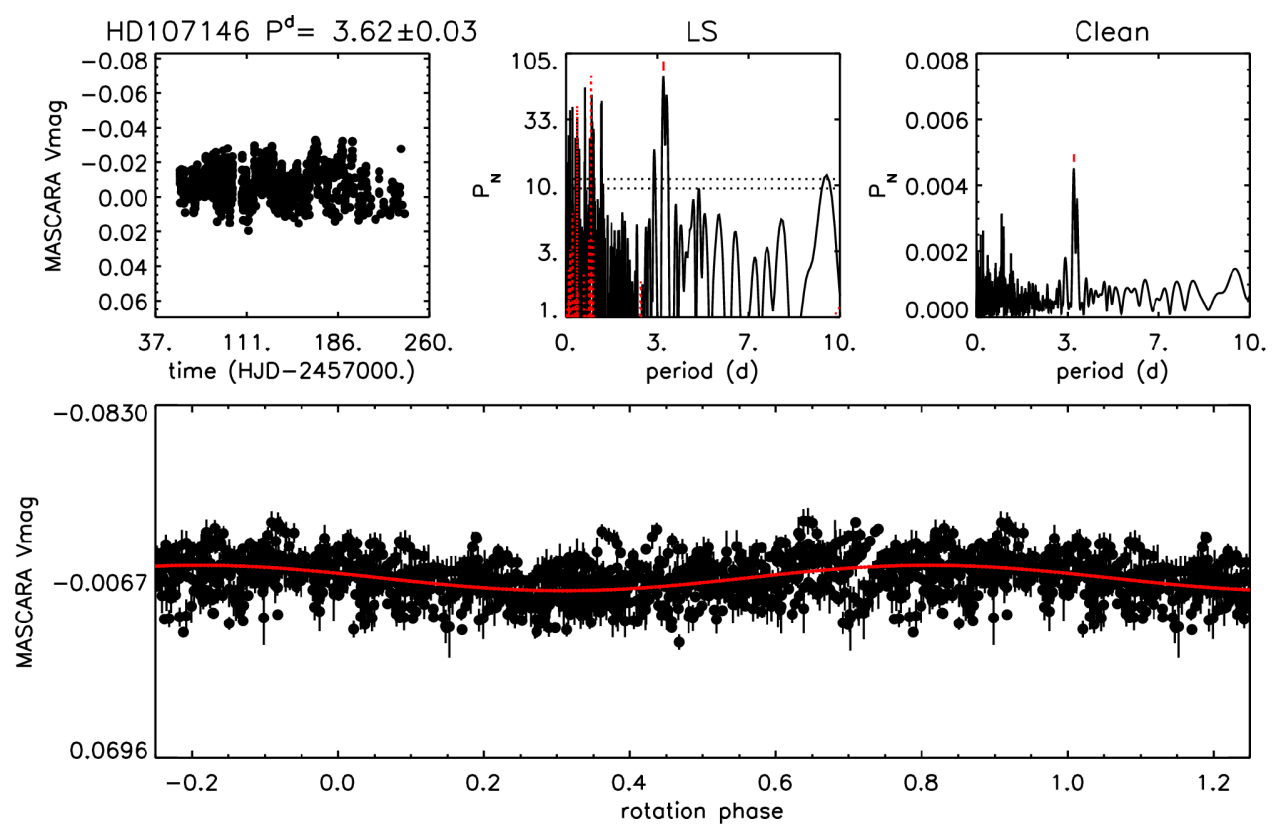

Figure 2. Results of the periodogram analysis of HD 107146 using MASCARA data. In the top left-hand panel we plot magnitudes versus heliocentric Julian date. In the top middle panel we plot the Lomb-Scargle periodogram with the spectral window function and power level corresponding to FAP $=1$ per cent overplotted (red dotted line), and we indicate the peak corresponding to the rotation period. In the top right-hand panel we plot the CLEAN periodogram. In the bottom panel we plot the light curve phased with the rotation period. The solid line represents the sinusoidal fit.

disc to be $7.7 \times 10^{-4}$. Recently, Marino et al. (2019) observed its disc using ALMA at $0.86 \mathrm{~mm}$ and detected, in the disc of planetesimals that extends from 50 to $140 \mathrm{au}$, a wide $\left(20_{-8}^{+10} \mathrm{au}\right)$ gap at a separation of $73 \pm 3$ au from the star. They also found for the disc an inclination of $65.4 \pm 0.9^{\circ}$ and a position angle of $100.0 \pm 0.9^{\circ}$. They concluded that if the gap was caused by a planet on a circular orbit, the planet would need to be less massive than $0.6 M_{\text {Jup }}$. Based on a possible asymmetry in the disc, they also proposed that the gap could have been carved due to secular resonance caused by two planets interior to the disc. In this context, they also defined different possible masses for these two objects.

Because this system has a bright debris disc, it has been the target of a large number of direct-imaging surveys (e.g. Janson et al. 2013; Biller et al. 2013; Vigan et al. 2017). In particular, Biller et al. (2013), adopting an age of $100 \mathrm{Myr}$ and exploiting NICI near-infrared observations and the AMES-COND (Allard et al. 2003) evolutionary models, were able to set mass limits of $\sim 10 M_{\text {Jup }}$ at a separation of $0.5 \operatorname{arcsec}(\sim 10.8 \mathrm{au}), \sim 4 M_{\text {Jup }}$ at a separation of $1 \operatorname{arcsec}(\sim 21.5 \mathrm{au})$, and less than $2 M_{\text {Jup }}$ at separations larger than $2 \operatorname{arcsec}(\sim 43 \mathrm{au})$.

\subsection{HD 107146}

HD 107146 is a G2V (Harlan \& Taylor 1970) star at a distance from the Sun of $27.47 \pm 0.03$ pc (Gaia Collaboration 2016, 2018).

\subsubsection{Spectroscopic analysis}

We exploited one FEROS spectrum (with a median SNR per pixel of 183) available from the ESO archive and obtained on 2018 April 30 under the open-time programme 0101.A-9012(A) (PI R. Launhardt). Following the same approach described in Section 2.1.1, we obtained $T_{\text {eff }}=5933 \pm 53 \mathrm{~K}, \log g=4.56 \pm 0.09 \mathrm{dex}$, microturbulent velocity $1.19 \pm 0.08 \mathrm{~km} \mathrm{~s}^{-1}$, and $[\mathrm{Fe} / \mathrm{H}]=+0.01 \pm 0.03 \mathrm{dex}$.
Broad-band colours $\left(B-V, V-I, B_{\mathrm{p}}-R_{\mathrm{p}}\right.$, and $\left.V-K\right)$ are consistent with the spectroscopic estimate of about $5900 \mathrm{~K}$ obtained from Pecaut \& Mamajek's (2013) tables.

\subsubsection{Stellar age}

HD 107146 was observed as a target of the MASCARA (Multi-site All-Sky CAmeRA; Burggraaff et al. 2018) project. Observations were collected from 2015 February until 2016 April, in two consecutive seasons for a total of 7188 magnitude measurements, with a cadence of about 1 measurement every $10 \mathrm{~min}$. After removal of outliers at the $3 \sigma$ level, we binned the data with a bin width of $1 \mathrm{~h}$ and were left for the subsequent analysis with 1716 average magnitudes with an average precision of $\sigma=0.0045$ mag. Both Lomb-Scargle and CLEAN periodogram analyses revealed the same rotation period within the uncertainties in the complete time series as well as in the single-observation seasons. In Fig. 2, we summarize the results for the first observation season whose monitoring turned out to be more homogeneous with no intra-season gaps. We found a rotation period of $P=3.62 \pm 0.03 \mathrm{~d}$ with a false alarm probability (FAP) $<1$ per cent and an amplitude of the magnitude rotational modulation $\Delta V=0.01 \mathrm{mag}$.

A comparison with the rotation period distribution of Pleiades members from Rebull et al. (2016) shows the HD 107146 period to fit very well into a $125 \mathrm{Myr}$ period distribution (assuming $V=7.04 \mathrm{mag}$ and $K=5.54 \mathrm{mag}, V-K=1.5 \mathrm{mag}$, and $P=3.62 \mathrm{~d}$ ). Adopting the rotation-age relations by Mamajek \& Hillenbrand (2008) we derive for HD 107146 an age of $156 \mathrm{Myr}$.

The activity indicators yield consistent results: the chromospheric emission $\left(\log R_{\mathrm{HK}}=-4.34\right.$; Wright et al. 2004) is within the distribution of the Pleiades, while the X-ray emission $\left(\log L_{\mathrm{X}} / L_{\mathrm{bol}}=\right.$ -4.33 ) is slightly below the Pleiades locus. The corresponding ages using the Mamajek \& Hillenbrand (2008) calibrations are 189 and $226 \mathrm{Myr}$, respectively. 
Table 1. Characteristics of the SPHERE observations presented in this work. In column 1 we list the observing night; in column 2 we list the target name. In column 3 and 4 we report the SPHERE observing mode and the coronagraph used, respectively. In columns 5 and 6 we list the number of data cubes, the number of frames for each data cube, and the exposure time for each frame for observation with IFS and with IRDIS, respectively. In column 7 we report the total rotation of the field of view (FOV) during each observation. In columns 8, 9, and 10 we report the median values of seeing, coherence time, and wind speed during the observations.

\begin{tabular}{|c|c|c|c|c|c|c|c|c|c|}
\hline Date & Target & Obs. mode & Coronagraph & Obs. IFS & Obs. IRDIS & $\begin{array}{c}\text { FOV rot. } \\
\left(^{\circ}\right)\end{array}$ & $\begin{array}{l}\text { seeing } \\
(\operatorname{arcsec})\end{array}$ & $\begin{array}{c}\tau_{0} \\
(\mathrm{~ms})\end{array}$ & $\begin{array}{l}\text { wind } \\
\left(\mathrm{m} \mathrm{s}^{-1}\right)\end{array}$ \\
\hline 2018 Jan 27 & HD 92945 & IRDIFS & N_ALC_YJH_S & $16 \times 6 ; 48$ & $16 \times 6 ; 48$ & 121.3 & 0.80 & 5.6 & 6.63 \\
\hline 2015 Apr 9 & HD 107146 & IRDIFS & N_ALC_YJH_S & $16 \times 5 ; 32$ & $16 \times 10 ; 16$ & 14.9 & 1.66 & 3.8 & 0.80 \\
\hline 2016 Mar 19 & HD 107146 & IRDIFS & N_ALC_YJH_S & $16 \times 5 ; 32$ & $16 \times 10 ; 16$ & 15.7 & 1.00 & 2.1 & 4.35 \\
\hline
\end{tabular}

We measured on the FEROS spectrum a Li equivalent width of $125.3 \pm 2.0 \mathrm{~m} \AA$ basically identical to the determination by Wichmann, Schmitt \& Hubrig (2003) (125 mA). This collocates HD 107146 within the lower envelope of the Pleiades distribution.

Moreover, the search for an association between HD 107146 and young moving groups did not give any result. We also searched the Gaia DR2 archive to identify possible comoving objects within $1^{\circ}$ from the star without identifying any.

On the basis of rotation period, level of chromospheric and coronal activity, and lithium content, the age of HD 107146 appears to be comparable to or slightly older than the Pleiades age. Also in this case we calculated a weighted combination of the available indicators following Desidera et al. (2015), and adopted an age of $150 \mathrm{Myr}$ with limits between 120 and $200 \mathrm{Myr}$.

\subsubsection{Mass, radius, and inclination}

The stellar mass derived using the same method used for HD 92945 as described in Section 2.1.3 is $1.06 \pm 0.01 \mathrm{M}_{\odot}$. A stellar radius of $0.94 \pm 0.02 R_{\odot}$ is also derived in the fit. Coupling this with the observed rotation period and the projected rotational velocity $v \sin i=5.0 \pm 0.5 \mathrm{~km} \mathrm{~s}^{-1}$ (Nordström et al. 2004; Valenti \& Fischer $2005)$, we infer an inclination of $22.4^{\circ} \pm 5.5^{\circ}$. This inclination is well aligned with the inclination estimated for the disc (see below) within the error bars.

\subsubsection{The debris disc}

The debris disc was first discovered by Silverstone (2000) using IRAS observations at $60 \mu \mathrm{m}$ while observations at submillimetre wavelengths confirmed the presence of dust (Williams et al. 2004). Later, the disc was resolved thanks to HST observations (Ardila et al. 2004; Ertel et al. 2011), revealing a disc extending up to a separation of $\sim 160$ au and with a brightness peak at 120 au. The infrared fractional luminosity of the disc was estimated to be $1.07 \times 10^{-3}$ (Ertel et al. 2011). Spitzer observations at 24 and $70 \mu \mathrm{m}$ allowed the determination of the presence of a warm dust belt in the system with a temperature of $\sim 120 \mathrm{~K}$ and located at a separation of 515 au from the star (Morales et al. 2011; Kennedy \& Wyatt 2014). Using ALMA data at $1.25 \mathrm{~mm}$, Ricci et al. (2015) found that the disc extends from 30-150 au from the star but with a decrease of brightness at intermediate radii that, in their interpretation, could be due to the presence of an Earth-mass planet orbiting at 80 au from the star. Finally, Marino et al. (2018) observed HD 107146 with ALMA at 0.86 and $1.1 \mathrm{~mm}$ and found that the planetesimal belt extends from 40-140 au and identified a circular gap at separations from $60-100$ au with a drop in emission by $\sim 50$ per cent. Finally, they were able to constrain the disc inclination to $19.3 \pm 1.0^{\circ}$ and the position angle to $153.0 \pm 3.0^{\circ}$ and concluded that either multiple low-mass planets or a single planet migrating through the disc were needed to make a wide gap that is only 50 percent empty.

\section{OBSERVATIONS AND DATA REDUCTION}

\subsection{Direct-imaging data}

HD 92945 was observed during the night of 2018 January 27 in the context of the SHINE survey (Chauvin et al. 2017; Vigan et al. 2020) with SPHERE operating in IRDIFS observing mode, i.e. with IFS (Claudi et al. 2008) observing in the $Y$ and $J$ spectral bands between 0.95 and $1.35 \mu \mathrm{m}$ and IRDIS (Dohlen et al. 2008) in the $H$ spectral band with the $\mathrm{H} 23$ filter pair (wavelength $\mathrm{H} 2=1.593 \mu \mathrm{m}$; wavelength $\mathrm{H} 3=1.667 \mu \mathrm{m}$; Vigan et al. 2010).

HD 107146 was observed in two different epochs in the context of the open-time programme 095.C-0374(A) (PI L. Ricci). The two epochs were acquired on 2015 April 9 and 2016 March 19, respectively. In both cases the observations were carried out with SPHERE operating in the IRDIFS observing mode. The observations for both targets were performed in pupil-stabilized mode to be able to implement angular differential imaging (ADI; Marois et al. 2006). The main characteristics of all the observing nights are listed in Table 1.

For all the observations, frames with satellite spots symmetric with respect to the central star were obtained before and after the coronagraphic sequences. This allowed determination of the position of the star behind the coronagraphic focal plane mask and accurate recentring of the data. Furthermore, to allow correct calibration of the flux of companions, images with the star not behind the coronagraph were taken. In these cases, the use of an appropriate neutral density filter was mandatory to avoid saturation of the detector.

The data were reduced through the SPHERE Data Center (Delorme et al. 2017) that performed the requested calibrations, both for IFS and IRDIS, using the data-reduction and handling (DRH; Pavlov, Feldt \& Henning 2008) pipeline. On the reduced data we then applied speckle subtraction algorithms using both TLOCI (Marois et al. 2014) and principal-component analysis (PCA; Soummer, Pueyo \& Larkin 2012) as implemented in the SPHERE consortium pipeline called SpeCal (spectral calibration; Galicher et al. 2018).

\subsection{Radial-velocity data}

To complement the direct-imaging mass limits at lower separations from the star we retrieved archival RV data for both stars. For HD 92945 we used the ESO archive data obtained using the HARPS spectrograph (Lovis et al. 2006) operating at the $3.6 \mathrm{~m}$ telescope at La Silla. These data were obtained in the context of the open-time programmes 074.C-0037(A) (PI E. Guenther), 075.C-0202(A) (PI 


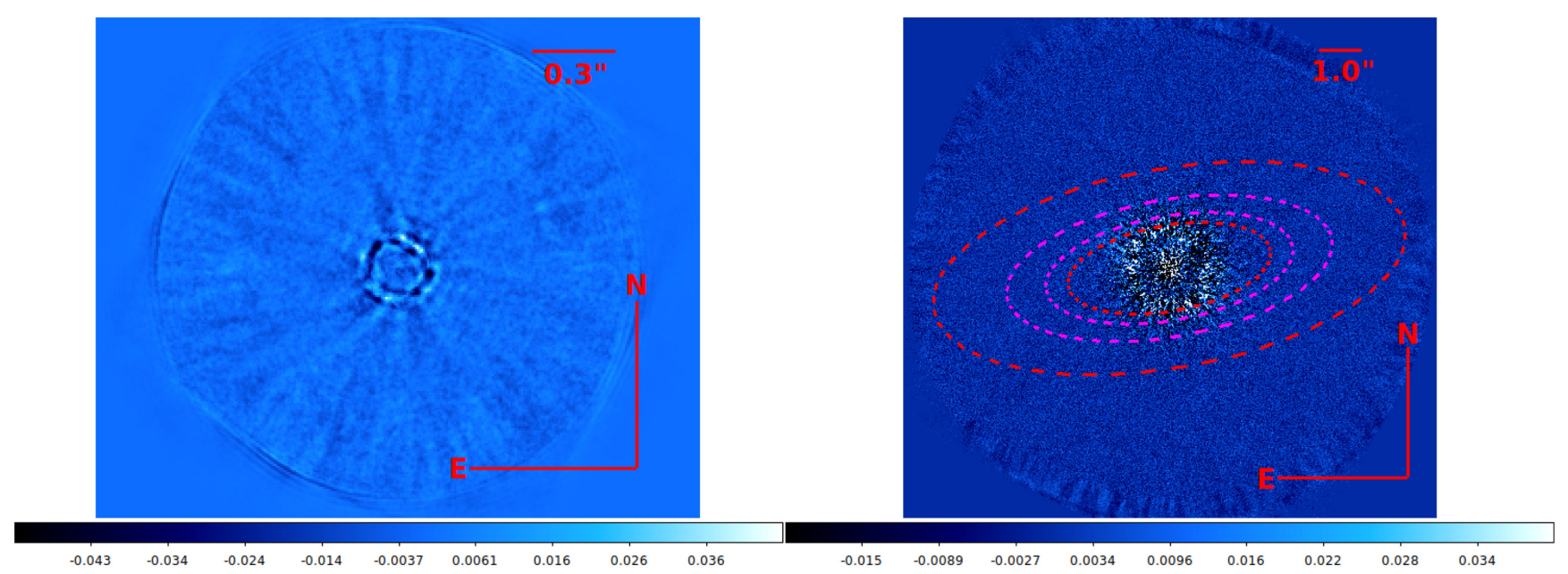

Figure 3. Final images obtained for HD 92945 using IFS data (left) and IRDIS (right). In both cases the data were reduced using PCA subtracting 100 and 25 principal components for IFS and IRDIS, respectively. On the IRDIS image we also plotted dashed lines indicating the position of the outer belt of the disc (red lines) and of the gap (magenta lines). All these lines are outside the FOV of IFS.

E. Guenther), and 192.C-0224(B) (PI A.-M. Lagrange). The data were reduced using the official HARPS pipeline (DRS v. 3.5), which delivers the RV and full width at half maximum (FWHM) measured on each cross-correlation function (CCF). The mask used is G2. We gathered a total of $28 \mathrm{RVs}, 10$ of them being taken in 2005, the remaining ones in 2016-17. Some of the data were then taken after an instrument upgrade in 2015 June (Lo Curto et al. 2015) and display an offset toward larger RVs with respect to the pre-upgrade data. This offset was applied before combining data. The typical precision for these observations was of the order of $1 \mathrm{~m} \mathrm{~s}^{-1}$.

RV data for HD 107146 covering the epochs from 2007-2015 were obtained using the SOPHIE high-resolution echelle spectrograph fibre-fed from the Cassegrain focus of the $1.93 \mathrm{~m}$ telescope at the Haute-Provence Observatory (OHP) in France (Bouchy \& Sophie Team 2006). SOPHIE is installed in a temperature-stabilized environment, and the dispersive elements are kept at constant pressure to provide high-precision radial velocities (Perruchot et al. 2008). Since 2011 June, a new fibre scrambler has provided a significant improvement to the spectrograph illumination stability, leading to a precision gain of a factor of $\sim 6$ (Perruchot et al. 2011; Bouchy et al. 2013). The spectra were reduced and extracted using the SOPHIE pipeline (Bouchy et al. 2009), and the resulting wavelengthcalibrated 2D spectra were correlated using a numerical binary mask corresponding to spectral type G2 to obtain the radial-velocity measurement (Baranne et al. 1996). In this case the typical precision was of the order of $2 \mathrm{~m} \mathrm{~s}^{-1}$.

\subsection{Proper-motion data}

The presence of a companion can be inferred from studying the anomalies of the proper motion of the host star. These anomalies are obtained comparing the long-term proper-motion vector derived comparing data from the Hipparcos (van Leeuwen 2007) and Gaia DR2 catalogues and the instantaneous proper-motion values at both the Hipparcos and Gaia DR2 epochs. Deviations between these vectors at SNR levels higher than three are indicative of the presence of a companion. Even in the case of no evidence of a low-mass companion, these data can still be used to define constraints on the mass of a companion, especially at low separations from the host star where the limits from direct imaging are less effective or totally absent due to the presence of the coronagraph. In recent work, Kervella et al. (2019) calculated the PMa for a sample of 6741 stars at a distance less than $50 \mathrm{pc}$ from the Sun. Both HD 92945 and HD 107146 were part of this sample and we can then exploit these results in combination with the limits from direct imaging and from $\mathrm{RV}$ to constrain the presence of companions around these systems. While for the case of HD 92945 they did not find any evidence of a companion, for HD 107146 they found an SNR of 3.42 on the PMa, strongly indicative of the presence of a companion. We will discuss in Section 5 the implications of this result.

\section{RESULTS}

\subsection{HD 92945}

In Fig. 3 we display the final images obtained for HD 92945 using both IFS (left) and IRDIS (right). We have overplotted on the IRDIS image dashed red lines to indicate the position of the disc and dashed magenta lines to indicate the position of the gap as indicated by Marino et al. (2019). The same positions were, however, outside the field of view (FOV) of IFS. No clear detection of possible candidate companions is in the FOV of both instruments. Also, the disc is undetected as expected due its low surface brightness typical for a $\sim 100$ Myr old debris disc and ADI self-cancellation effects (Milli et al. 2012). We then calculated the $5 \sigma$ luminosity contrast as a function of the separation from the star adopting the technique presented in Mesa et al. (2015) and corrected for the small sample statistic following the method described by Mawet et al. (2014). The contrast in magnitude derived with this method for both IFS and IRDIS is displayed in Fig. 4. With IFS, we obtain a contrast of the order of 15 mag at separations larger than 0.5 arcsec, while IRDIS allows us to obtain contrasts better than 16 mag at separations larger than $\sim 1.5$ arcsec. These contrast estimates assume that the disc is optically thin, which is true for this system and for debris discs in general.

Adopting this contrast and exploiting the AMES-COND evolutionary models, we defined the mass limits for possible companions around HD 92945 adopting both the minimum (120 Myr) and the maximum (250 Myr) ages proposed for this system. The separation on the disc plane was considered in this case adopting an inclination 


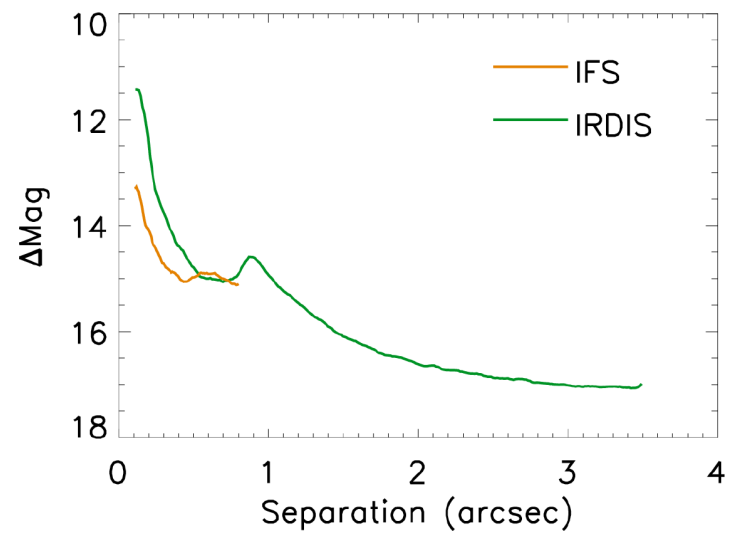

Figure 4. Magnitude contrast versus projected separation in the region around HD 92945 for both IFS (orange line) and IRDIS (green line).

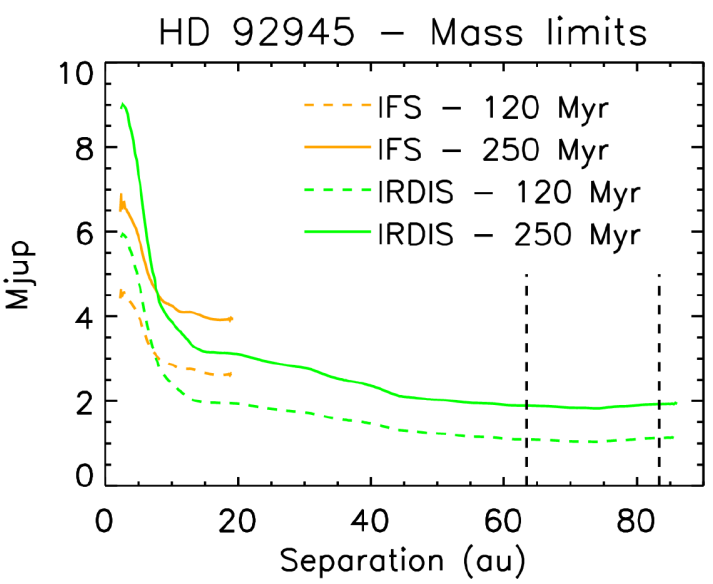

Figure 5. Mass limits obtained for HD 92945 using the AMES-COND models for both IFS (orange lines) and IRDIS (green lines). The dashed lines represent the mass limits obtained assuming an age of $120 \mathrm{Myr}$ for the system; the solid lines are obtained assuming an age of $250 \mathrm{Myr}$. The two dashed vertical lines correspond to the expected position of the gap.

of $65.4^{\circ}$ and a position angle of $100.0^{\circ}$ (Marino et al. 2019). Using this information we calculated for each position in the image both the mass limits and the deprojected separation from the star. The median value of the mass for positions at the same separation from the star was assumed as the mass limit at that separation. The results obtained from this procedure are displayed in Fig. 5 where the dashed lines represent the mass limits obtained for the 120 Myr age case while the solid lines represent the $250 \mathrm{Myr}$ case. Also, in this figure we distinguish between the IFS case, represented by the two orange lines, and the IRDIS case, represented by the two green lines. The two dashed vertical lines, finally, represent the expected position of the gap in the disc. From our results, we can see that at this position the mass limits are between 1 and $2 M_{\text {Jup }}$ depending on the age of the system. These limits are still higher than the maximum mass of $0.6 M_{\text {Jup }}$ evaluated for a single planet carving the gap proposed by Marino et al. (2019). Nevertheless, these limits are useful to rule out the presence of massive companions interior or exterior to the disc. As for the solution with two inner planets proposed by Marino et al. (2019) to explain the gap (see e.g. their fig. 8), our data are able in this case to further constrain the possible configurations proposed in that paper.

\subsection{HD 107146}

In Fig. 6 we display the final images obtained both for IFS (left) and IRDIS (right) for the HD 107146 data obtained during the night of 2016 March 19. We also overplotted on the IRDIS image red dashed lines to indicate the position of the disc and magenta dashed lines to indicate the position of the gap using the values obtained by Marino et al. (2018). These lines are outside the IFS FOV. As in the case of HD 92945 we are not able to retrieve any signal from the disc. Also in this case, this is expected due to the low luminosity at the estimated age of the system and to the moderately low inclination of the disc with respect to the line of sight. In this case, however, a point source is clearly detected at a separation larger than 5 arcsec and a position angle of $\sim 213^{\circ}$ south-west of the star in both the observing epochs (see Table 2 for precise astrometric data). In Fig. 7 we display the comparison of the relative astrometric positions in the two epochs listed in Table 2. The relative position of the candidate in the second epoch (violet diamond) is very similar to its expected position if it was a background object (black squares), while we would expect a reduced shift from the relative position of the first epoch (green square) if it were a bound object. From this analysis we then conclude that the candidate is very likely a background object.

Following the same method described in Section 4.1 for HD 92945 we calculated the luminosity contrast in the region around HD 107146. The results of this procedure are shown in Fig. 8 both for IFS (orange line) and IRDIS (green line). From IFS we obtain a contrast better than 14 mag at separations larger than 0.5 arcsec while IRDIS allows us to obtain a contrast better than 16 mag at separations larger than 2 arcsec.

In Fig. 9 we display the results of the procedure that, using the AMES-COND evolutionary models and assuming for the disc an inclination of $19.3^{\circ}$ and a position angle of $153^{\circ}$ (Marino et al. 2018), converts these contrast limits into mass limits adopting both a minimum age of $120 \mathrm{Myr}$ (dashed lines) and a maximum age of $200 \mathrm{Myr}$ (solid lines). The two dashed vertical lines represent the expected position of the gap in the disc. In the gap, the mass limits that we obtain from our data are between 1 and $2.5 M_{\text {Jup }}$. At lower separations, we reach limits slightly lower than $5 M_{\text {Jup }}$ between 20 and 50 au. Interior from 20 au the mass limits are typically larger than $5 M_{\text {Jup }}$.

\section{DISCUSSION}

\subsection{Comparison with dynamical models}

To further check how the limits that we obtained can help to constrain the structures of these systems we compared them with the results of dynamical models following the method devised in Lazzoni et al. (2018). This method derives analytical tools to link the extension of gaps detected in debris discs with the masses, semimajor axes, and eccentricities of putative planets responsible for the gaps. The main assumption underneath this formulation is that a planet sweeps dust particles from a region around its orbit called the chaotic zone. The width of the chaotic zone is directly proportional to the mass, the semimajor axis, and the eccentricity of the planet. When more than one planet is present in the gap, not only does the planetdisc interaction have to be taken into account, but the planet-planet interaction must also be treated. Since a stable system is needed to preserve the debris disc, we included this condition in deriving the multiplanetary architecture. Moreover, to avoid further degeneracies in masses and semimajor axes, we assumed planets with equal mass 


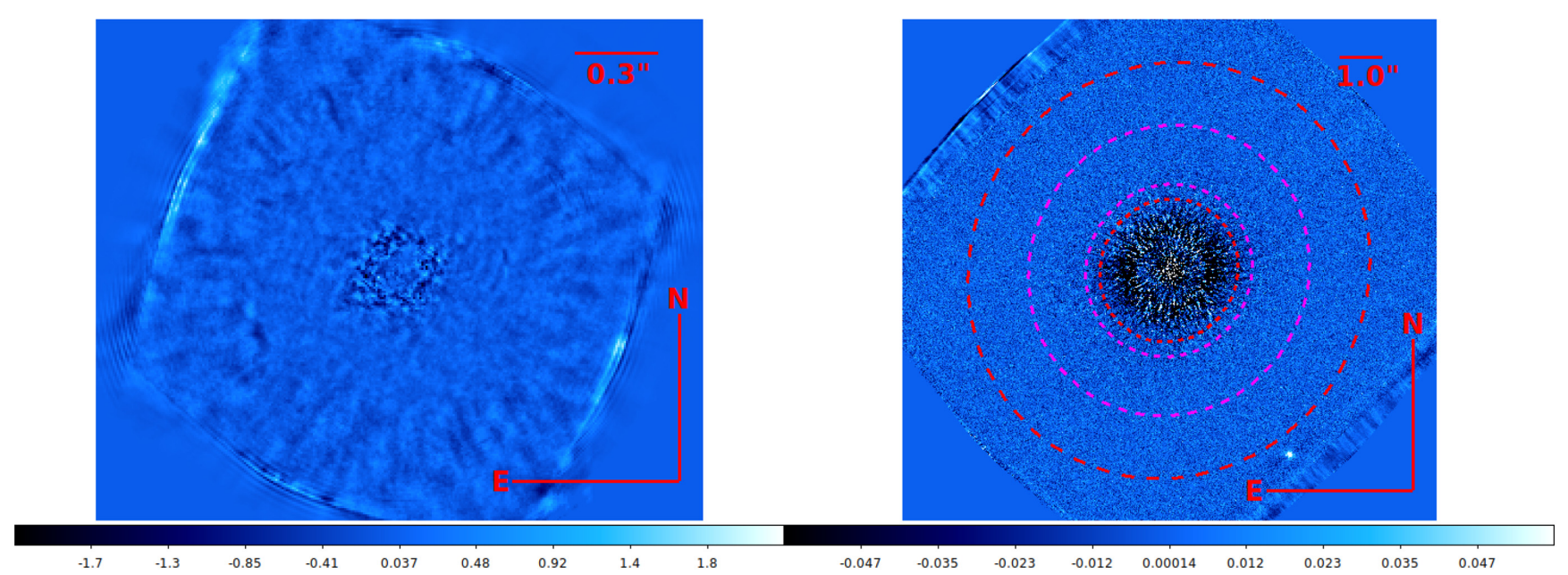

Figure 6. Final images obtained for HD 107146 using IFS data (left) and IRDIS (right) for the 2016 March 19 epoch. In both cases the data were reduced using PCA subtracting 100 and 25 principal components for IFS and IRDIS, respectively. We also plotted dashed lines to indicate the position of the outer belt of the disc (red lines) and of the gap (magenta lines) as defined in Marino et al. (2018). These lines are outside the IFS FOV.

Table 2. Astrometric positions relative to the host star of the candidate companion detected around HD 107146.

\begin{tabular}{lcccc}
\hline Obs. date & $\Delta$ RA (mas) & $\Delta$ Dec. (mas) & Separation $(\mathrm{mas})$ & PA $(\mathrm{deg})$ \\
\hline 2015 Apr 9 & $-2948.58 \pm 4.00$ & $-4487.17 \pm 4.00$ & $5369.25 \pm 5.66$ & $213.3 \pm 0.5$ \\
2016 Mar 19 & $-2811.68 \pm 4.00$ & $-4315.67 \pm 4.00$ & $5150.78 \pm 5.66$ & $213.1 \pm 0.5$ \\
\hline
\end{tabular}

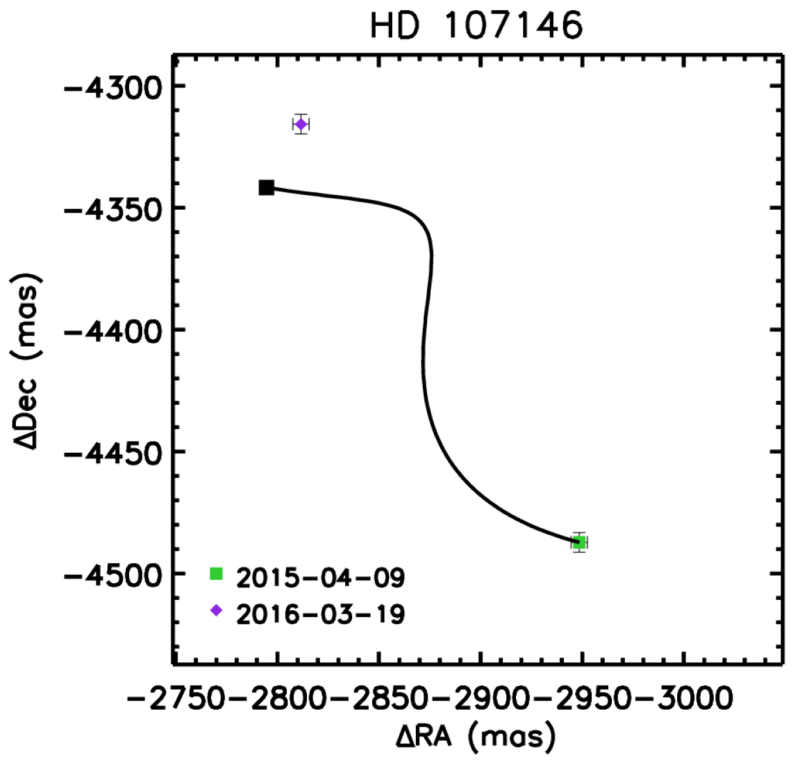

Figure 7. Relative astrometric positions of the candidate companion of HD 107146 with respect to the host star in the two observing epochs. The green square represents the relative position of the candidate companion at the first observing epoch. The violet diamond represents the relative position of the candidate companion in the second observing epoch. The solid black line represents the expected course of the companion if it were a background object. The black square at the end of the line represents the expected position at the epoch of the second observation in this latter case.

and as close as possible to still have a marginally stable system. We will refer to this last assumption as the max packing condition.

Regarding the circular orbit case, the planet-disc interaction is ruled by the equation derived in Morrison \& Malhotra (2015); see

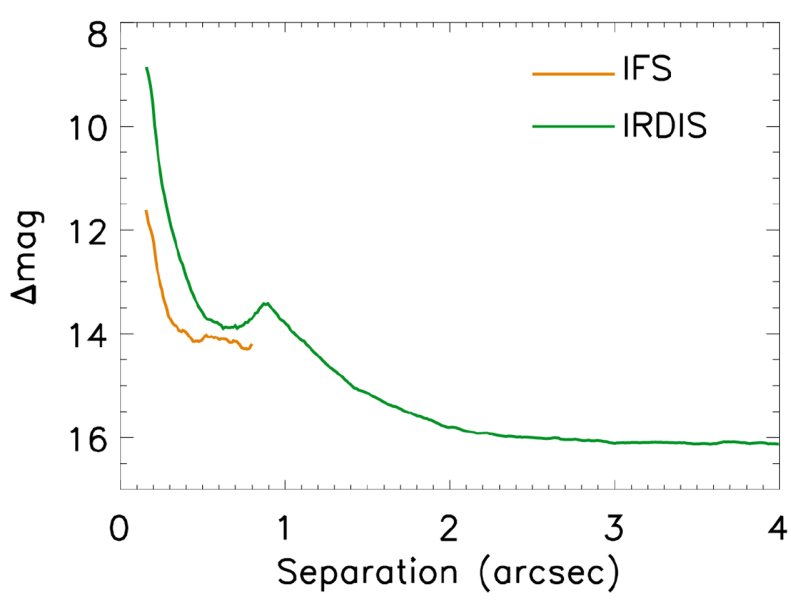

Figure 8. Magnitude contrast versus projected separation in the region around HD 107146 for both IFS (orange line) and IRDIS (green line).

also equations (3) and (4) in Lazzoni et al. (2018) for more details. Moreover, for two planets on circular orbits the stability criterion was derived from Gladman (1993) setting the eccentricities to zero and assuming equal masses to simplify the calculation as shown by equation (23) in Lazzoni et al. (2018). We also considered the case of three equal-mass planets. In this case the stability of the system is guaranteed by the Hill criterion coupled with $K$-parametrization curves derived by Marzari (2014) and also reported in equation (25) in Lazzoni et al. (2018).

We decided not to consider the case of eccentric orbits because the disc eccentricity for both systems is very low with an upper limit of 0.097 for HD 92945 (Marino et al. 2018) and 0.03 for HD 107146 (Marino et al. 2019). This should exclude the presence of higheccentricity embedded planets (Pearce \& Wyatt 2014, 2015). For all 


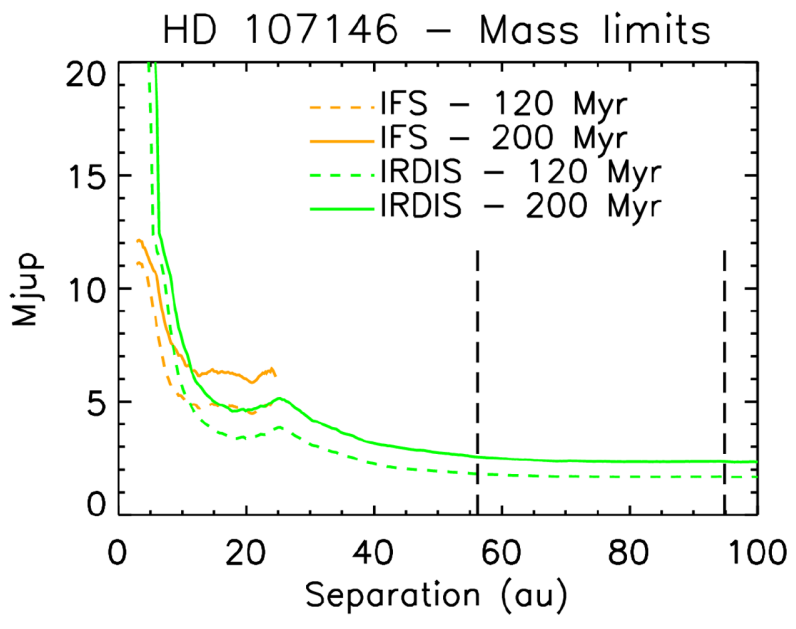

Figure 9. Mass limits obtained for HD 107146 using the AMES-COND models for both IFS (orange lines) and IRDIS (green lines). The dashed lines represent the mass limits obtained assuming an age of $120 \mathrm{Myr}$ for the system; the solid lines are obtained assuming an age of $200 \mathrm{Myr}$. The two dashed vertical lines correspond to the expected position of the gap.

the cases considered we assumed that the gap was completely devoid of dust particles. Other studies (e.g. Shannon et al. 2016) consider the gap as only marginally cleared. In such cases, the masses derived for the planets will be lower with respect to the ones derived in our analysis. We can thus consider our solutions as upper limits for each architecture considered. The results of this procedure are displayed in Fig. 10 both for HD92945 (left) and HD107146 (right).

Thanks to the procedure described above, we estimated that, in the case of HD 92945, a single planet on a circular orbit should have a mass of at most $0.3 M_{\text {Jup }}$ to carve the gap. This is slightly lower but still in good agreement with the upper limit of $0.6 M_{\text {Jup }}$ found by Marino et al. (2019). If the gap was carved by multiple planets, their masses could be even lower. Clearly, all these planets have masses well below the detection limit of around $1 M_{\text {Jup }}$ reachable with SPHERE or with any other current planet imager.

In the case of HD 107146 the gap could be caused by a single planet with a mass of at most $3.3 M_{\text {Jup }}$. This would allow the detection according to the mass limit that we obtained for this system. However, we have to stress that, as explained above, this value has to be regarded just an upper limit. Considering two or three planets would require masses of $0.47 M_{\text {Jup }}$ and $0.03 M_{\text {Jup }}$, respectively, for all the considered planets. This is well outside the detection capability of our observations.

\subsection{Comparison with limits from RV and PMa}

\subsubsection{SPHERE detection probability}

The Exoplanet Detection Map Calculator (EXO-DMC; Bonavita 2020 ) is the latest (and, for the first time, in PYTHON) rendition of MESS (Multi-purpose Exoplanet Simulation System; Bonavita et al. 2012), a Monte Carlo tool for the statistical analysis of direct-imaging survey results. In a similar fashion to its predecessors, the DMC combines information on the target stars with the instrument detection limits to estimate the probability of detection of a given synthetic planet population, ultimately generating detection probability maps.

For each star in the sample the DMC produces a grid of masses and physical separations of synthetic companions, then estimates the probability of detection given the provided detection limits. The default set-up uses flat distributions in log space for both the mass and semimajor axis but, in a similar fashion to its predecessors, the DMC allows for a high level of flexibility in terms of possible assumptions on the synthetic planet population to be used for the determination of the detection probability.

For each point in the mass/semimajor axis grid the DMC generates a fixed number of sets of orbital parameters. By default all the orbital parameters are uniformly distributed except for the eccentricity, which is generated using a Gaussian eccentricity distribution with $\mu=0$ and $\sigma=0.3$, following the approach by Hogg, Myers \& Bovy (2010) (see Bonavita, de Mooij \& Jayawardhana 2013 for details). For this reason we were able to properly take into account the effects of projection when estimating the detection probability using the contrast limits in Figs 4 and 8. The DMC in fact calculates the projected separations corresponding to each orbital set for all the values of the semimajor axis in the grid (see Bonavita et al. 2012 for a detailed description of the method used for the projection). This allows us to estimate the probability of each synthetic companion truly being in the instrument FOV and therefore being detected, if the value of the mass is higher than the limit.

In this specific case we chose to restrict the inclination and the longitude of the node of each orbital set to make sure that all companions in the population would lie in the same orbital plane as the disc. This is equivalent to assuming that there would be no strong misalignments $(\leq 5 \mathrm{deg})$ between the discs and possible embedded planets. A massive misaligned planet would in fact reorient the disc through secular interactions and the new mid-plane would be aligned with the planet. This would typically occur on time-scales shorter than the age of these systems (Pearce \& Wyatt 2014).

Moreover, since in both cases the disc does not appear to be particularly eccentric (Marino et al. 2018, 2019), which would be the case if the orbits of the embedded planets were (Pearce \& Wyatt 2014, 2015), we assumed a sigma of 0.1 for the eccentricity distribution.

In Fig. 11 we show the results for both targets. In order to take into account the uncertainty on the age values, two different DMC runs were performed for each target, using the minimum and maximum values of the mass limits, respectively.

\subsubsection{Comparison with available radial-velocity measurements}

While MESS was limited in its use to direct-imaging data, ${ }^{3}$ the DMC can also be used to draw similar constraints using other kind of data sets, including radial-velocity (RV) ones. Given the provided RV time series, the DMC uses the local power analysis (LPA) approach described by Meunier, Lagrange \& De Bondt (2012) to estimate, for each mass and separation in the grid, for what fraction of the generated orbital sets the signal generated by the companion would be compatible with the data.

Once the computation is completed, the code outputs a $2 \mathrm{D}$ detection probability map analogous to the ones produced with the DI module as well as a $1 \mathrm{D}$ detection limit showing the minimum value of the companion mass detectable with a given confidence level. The latter, which is roughly equivalent to extracting a specific detection probability contour from the 2D map, is the standard approach used to obtain RV detection limits in the past. The advantage of retaining

\footnotetext{
${ }^{3}$ With the exception of its MESS2 incarnation, which was designed to produce combined RV + DI detection probability maps (see Lannier et al. 2017 for details).
} 

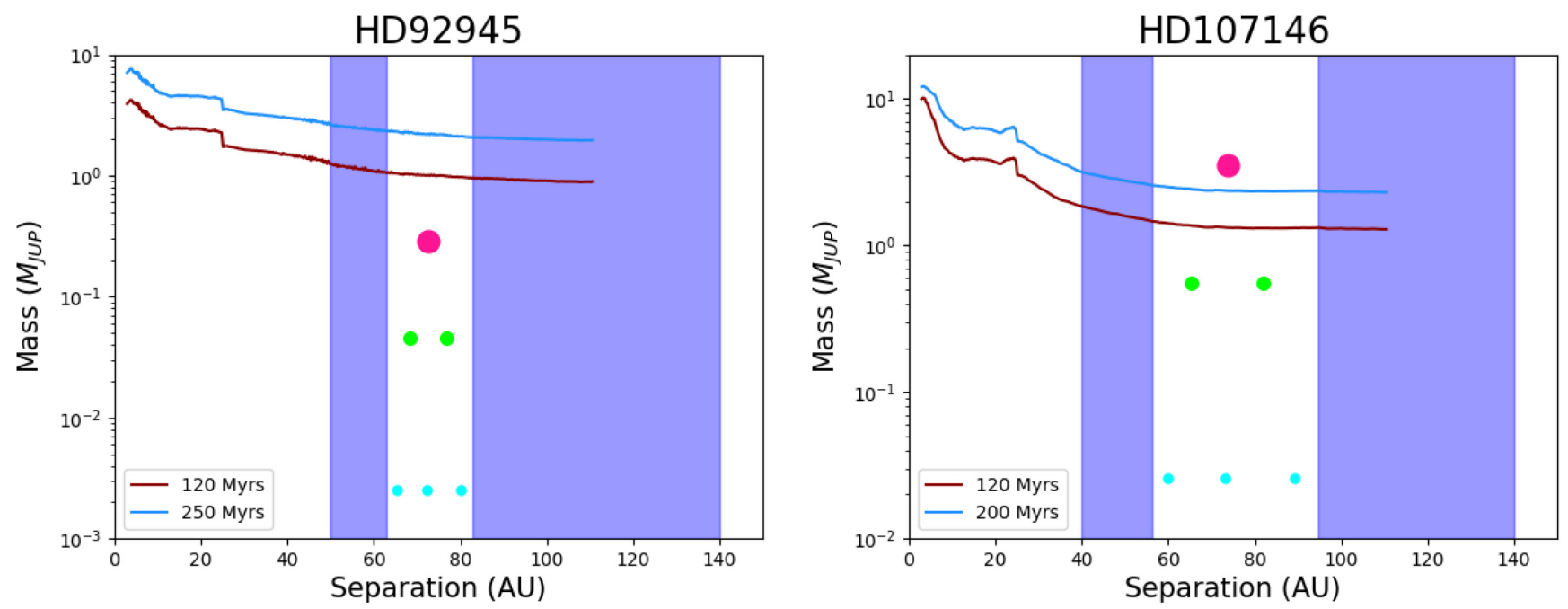

Figure 10. Upper limits of planetary masses from dynamical models considering one (magenta circle), two (green circles), or three (cyan circles) planets with circular orbits in the case of both HD 92945 (left) and HD 107146 (right). The shaded areas represent the positions of the disc with the gap positions also displayed. For comparison we also include the mass limit obtained through direct imaging.
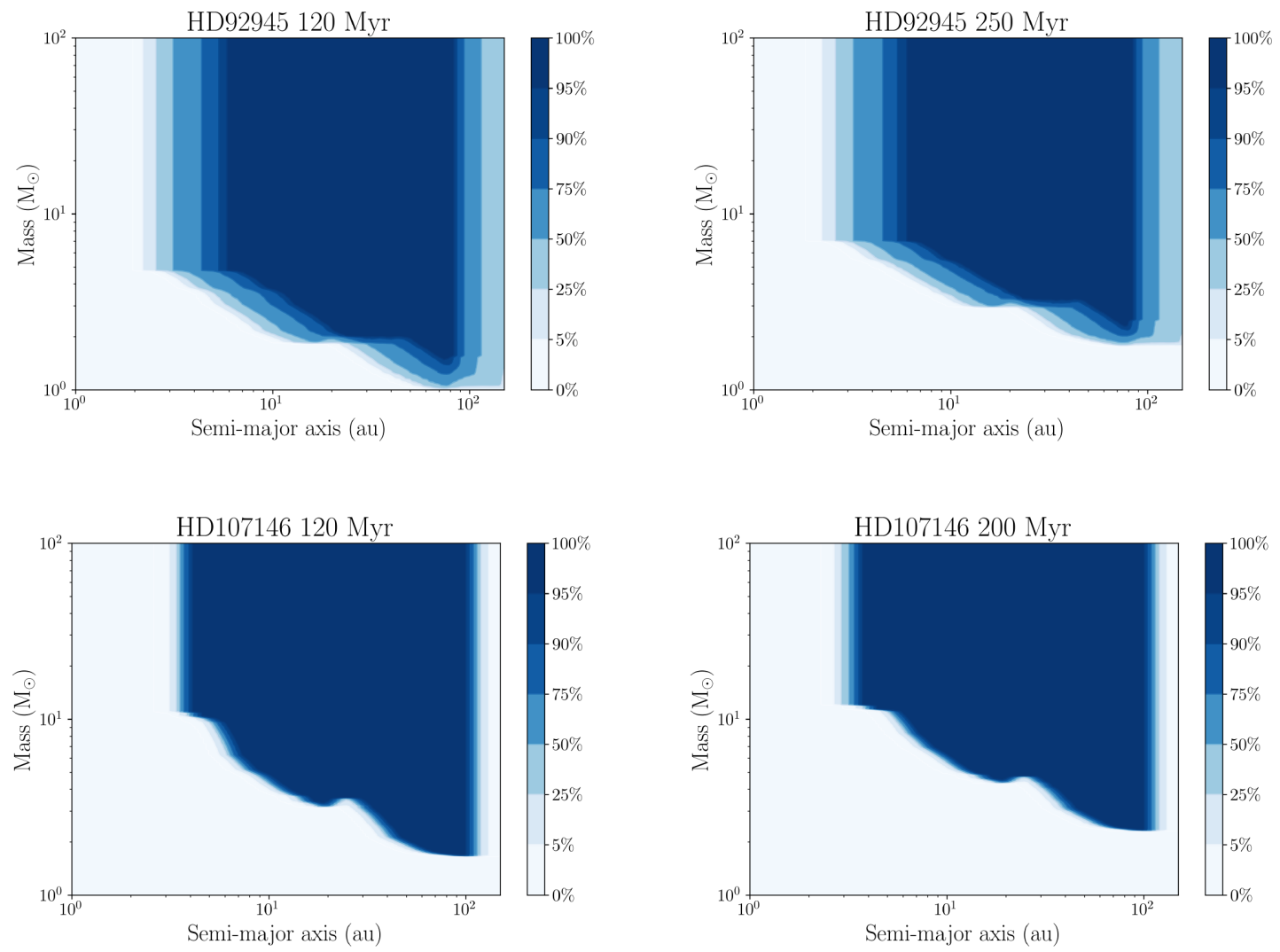

Figure 11. Detection probability maps, obtained with the DMC code, for HD 92945 (left) and HD 107146 (right). Given the uncertainty on the age of the targets, we show the results of the runs performed using the contrast limits converted to mass using both the minimum and maximum age estimates (upper and lower panels, respectively).

the $2 \mathrm{D}$ information is that the resulting RV probability map can then be easily combined with the output of the DI module. ${ }^{4}$

${ }^{4}$ One should note that the current version of DMC does not allow for a truly accurate estimate of the sensitivity achieved combining the two data sets, like the one performed in Lannier et al. (2017). The method used only allows for
We therefore run the RV-DMC on the available data for our two targets, using the same mass/semimajor axis grid as well as the same assumptions for the orbital parameter generation. The two sets of

a rough estimate of the combined probability. A new module analogous to MESS2 will be available in the future. 

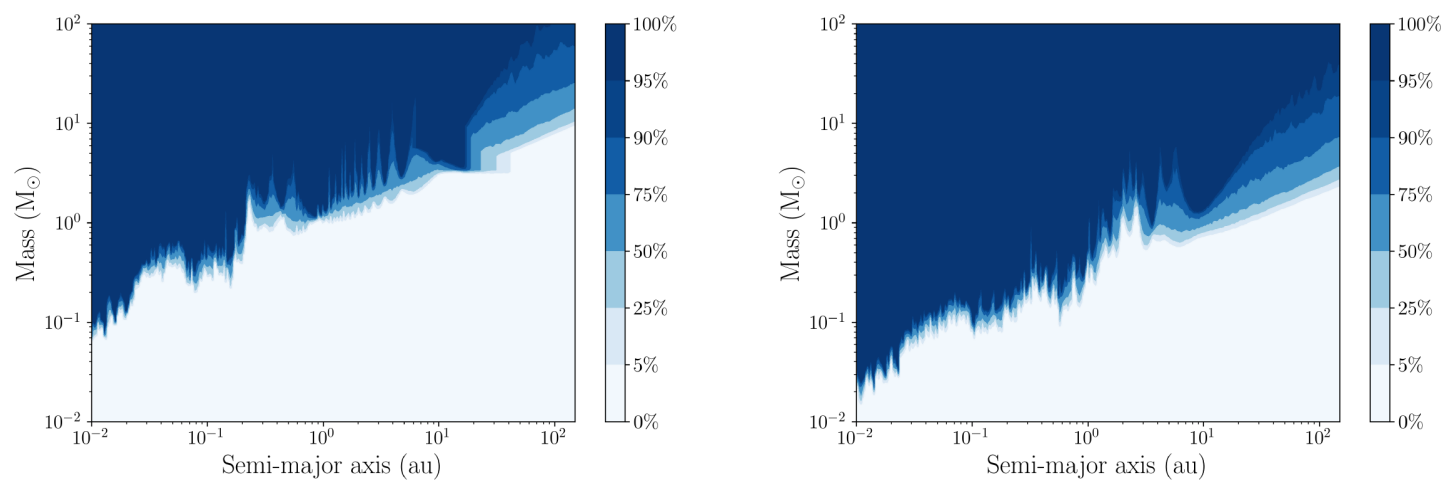

Figure 12. Combined RV + DI detection probability maps for HD 92945 (left) and HD 107146 (right), estimated considering the best value of the probability for each point in the grid (see Section 5.2.2). The values of the age for the magnitude-to-mass conversion of the DI limits in this case were set to $170 \mathrm{Myr}$ for HD 92945 and 150 Myr for HD 107146.

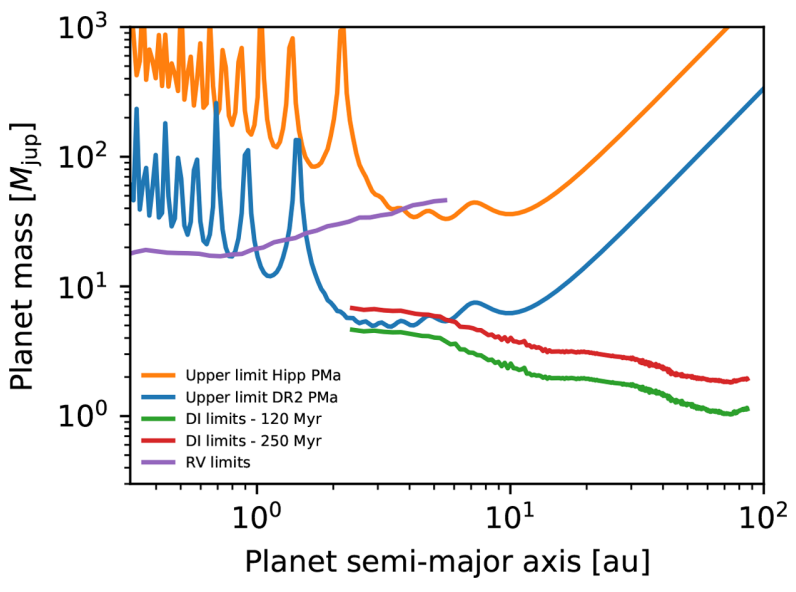

Figure 13. Upper limits in mass obtained from PMa measurements for the Hipparcos epoch (orange line) and for the Gaia DR2 epoch (blue line) for HD 92945 compared with the limits from the RV data (violet line, assuming a 95 per cent confidence level, see Section 5.2.2 for details) and from the DI data for an age of $120 \mathrm{Myr}$ (green line) and for an age of $250 \mathrm{Myr}$ (red line).

maps were then combined by considering, for each point in the grid, the best value of the probability. The resulting combined map, shown in Fig. 12, then contains the probability that a companion at a given mass and semimajor axis is detected using at least one of the two methods.

\subsubsection{Comparison with PMa results}

As explained in Section 3.3 PMa data are available for both targets and they can be used to calculate the mass of a possible object causing the anomaly as a function of the separation from the host star, exploiting equation (15) in Kervella et al. (2019). For HD 92945 no clear evidence of the presence of a companion emerges from these data. We can thus use these data just to put upper limits on the masses of possible companions. They are, however, comparable with those obtained through RV and DI at separations between 1 and 10 au only, while they are clearly worse at smaller and larger separations. This can be easily seen in Fig. 13 where the upper limits from the PMa measurements obtained using data from the Hipparcos and the Gaia DR2 epochs (orange and blue lines; labelled as upper limit Hipp PMa and upper limit DR2 PMa) are compared with mass

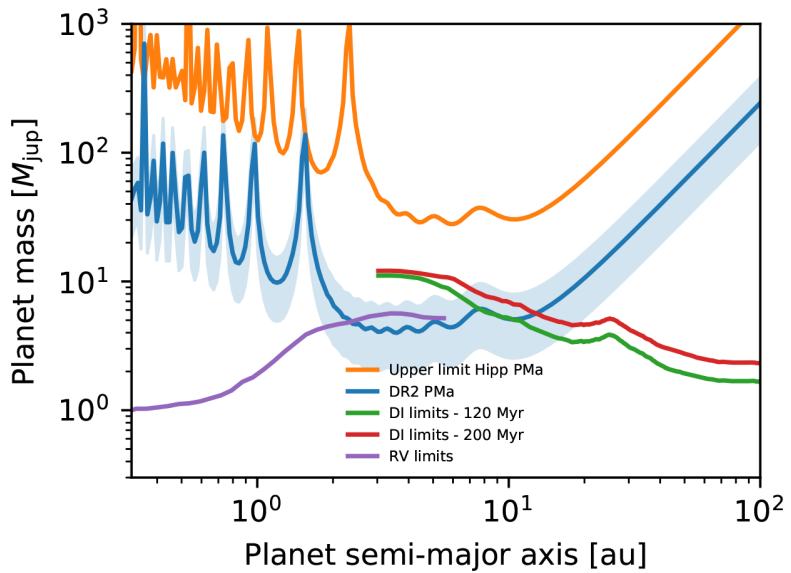

Figure 14. Mass as a function of the separation from the host star of the companion needed to explain the PMa measurement at the Gaia DR2 epoch for HD 107146 (blue line). The blue shaded area display the 99.7 percent confidence interval. The orange line represents the upper limits from PM measurements for the Hipparcos epoch while the violet line displays the mass limits from RV data (assuming a 95 percent confidence level; see Section 5.2.2 for details). The DI mass limits adopting an age of $120 \mathrm{Myr}$ are shown by the green line while the DI limits adopting an age of 200 Myr are shown by the red line.

limits from RV data (violet line) and from DI data (red and green lines).

There is a different situation in HD 107146, where a $3.4 \sigma$ PMa obtained at the Gaia DR2 epoch strongly suggests the presence of a companion. Using equation (15) in Kervella et al. (2019) and a Monte Carlo approach as in Marino et al. (2020) we can estimate the mass of the companion responsible for the PMa as a function of the semimajor axis. This is represented by the blue line in Fig. 14 and is labelled as DR2 PMa while the shaded blue area surrounding this line is the 99.7 per cent confidence interval. The 95 per cent 1D mass limits obtained with the RV module of EXO-DMC (violet line) combined with the mass limits from the DI data (red and green lines) allow us to exclude a large part of the possible masses and separations for this putative object. In this way, if it exists, it could only be at separations between $\sim 2$ and 7 au and with a mass of 2-5 $M_{\text {Jup }}$. Future imaging instruments operating at extremely large telescopes (ELT) should be able to image such a planet that will also be clearly detected through the next Gaia data releases. It has to be noted that the 
presence of an asteroid belt at a separation between 5 and 15 au has been inferred thanks to Spitzer data (Morales et al. 2011; Kennedy \& Wyatt 2014). If confirmed, the proposed companion would lie just inside the position of this belt and its presence could explain the formation of the belt with the pressure bump due to the presence of such a massive planet.

\section{CONCLUSION}

We have presented the results of the SPHERE observations of the stars HD 92945 and HD 107146. Both these stars are known to host a debris disc with an outer planetesimal belt containing a wide gap. Moreover, they share similar ages. Given the morphology of these gaps, it was proposed that these could have been carved by one or more planetary-mass companions, residing inside the gap for both systems (Ricci et al. 2015; Marino et al. 2018) or possibly interior to the disc for HD 92945 since its gap appears marginally asymmetric (Marino et al. 2019). The capability of putting strong constraints on the mass of possible companions around these objects can help us to better understand the formation mechanisms of these structures. Moreover, due to the paucity of gas in these discs (Marino et al. 2018, 2019), the problem of strong absorption that can be experienced with younger and gas-rich discs (e.g. the case of HD 163296; Mesa et al. 2019b) should be less important, making more reliable the limits obtained around these objects. On the other hand, the older ages of these objects with respect to systems hosting protoplanetary discs makes it harder to detect low-mass planets.

In any case, the mass limits that we obtain from our observations are much lower than those obtained before with previous DI observations. Indeed, for both the systems considered here, we were able to put very tight mass limits of $1-2 M_{\text {Jup }}$ according to the age considered for the system. Regarding the inner region with respect to the planetesimal belt, we can reach for both systems mass limits lower than 4-5 $M_{\text {Jup }}$ for the range of possible ages considered. These limits are enough to exclude some of the proposed configurations of inner planets that could explain the structure of the gap.

While the contrast obtained with SPHERE is still not enough to confirm or to exclude a large part of the range of possible companion masses that could explain the shape of the planetesimal belts around HD 92945 and HD 107146, the mass limits much tighter than those obtained in past surveys can help in limiting the possible configurations of these planetary systems. These data demonstrate the potential of direct imaging in the understanding of the structure of planetary systems. To fully exploit this potential, however, we will probably need to wait for future instrumentation to reach higher contrasts and larger spatial resolutions. As an example, the external planets responsible for carving the gap should be recoverable using the JWST NirCAM operating in coronagraphic observing mode in the wavelength range $3-5 \mu \mathrm{m}$, as at separations of some tens of au it should be able to image companions down to the Saturn mass (Beichman et al. 2012).

At low separations from the star, DI observations are not able to set tight mass limits. Moreover, the presence of the coronagraph hides the inner region of the planetary system. We thus used both PMa and RV data to obtain information on the inner regions of these systems. For HD 92945, no clear signal was obtained from PMa and we can just put upper limits that are, however, comparable to those obtained through RV data (some tens of $M_{\mathrm{Jup}}$ ) and through DI at higher separations. Much more interesting is the situation for HD 107146, where there is a tentative $3.4 \sigma$ PMa indicative of the presence of a companion. Coupling this result with the limits coming from RV and DI, we are able to constrain both the separation $(\sim 2-7 \mathrm{au})$ and the mass (2-
$5 M_{\text {Jup }}$ ) of such objects. We note here that future ELT coronagraphic instruments will possess the angular resolution required to detect such an object while the next Gaia releases should be able to further confirm its existence.

\section{ACKNOWLEDGEMENTS}

Based on observations collected at the European Organisation for Astronomical Research in the Southern Hemisphere under ESO programmes 095.C-0374(A), 1100.C-0481(D), 074.C-0037(A), 075.C0202(A), and 192.C-0224(B). Partly based on observations collected with the SOPHIE spectrograph on the $1.93 \mathrm{~m}$ telescope at the Observatoire de Haute-Provence (CNRS), France under programmes 07A.PNP.CONS, 08A.PNP.CONS, 12A.DISC.WATS, 13A.PNP.DELF, 14A.PNP.LAGR, 14B.PNP.LAGR. SPHERE is an instrument designed and built by a consortium consisting of IPAG (Grenoble, France), MPIA (Heidelberg, Germany), LAM (Marseille, France), LESIA (Paris, France), Laboratoire Lagrange (Nice, France), INAF-Osservatorio di Padova (Italy), Observatoire de Genève (Switzerland), ETH Zurich (Switzerland), NOVA (Netherlands), ONERA (France), and ASTRON (Netherlands) in collaboration with tESO. SPHERE was funded by ESO, with additional contributions from CNRS (France), MPIA (Germany), INAF (Italy), FINES (Switzerland), and NOVA (Netherlands). SPHERE also received funding from the European Commission Sixth and Seventh Framework Programmes as part of the Optical Infrared Coordination Network for Astronomy (OPTICON) under grant number RII3-Ct2004-001566 for FP6 (2004-2008), grant number 226604 for FP7 (2009-2012), and grant number 312430 for FP7 (2013-2016). We also acknowledge financial support from the Programme National de Planétologie (PNP) and the Programme National de Physique Stellaire (PNPS) of CNRS-INSU in France. This work has also been supported by a grant from the French Labex OSUG@2020 (Investissements d'avenir - ANR10 LABX56). The project is supported by CNRS, by the Agence Nationale de la Recherche (ANR14-CE33-0018). It has also been carried out within the frame of the National Centre for Competence in Research PlanetS supported by the Swiss National Science Foundation (SNSF).

This work has made use of data from the European Space Agency (ESA) mission Gaia (https://www.cosmos.esa.int/gaia), processed by the Gaia Data Processing and Analysis Consortium (DPAC, https: //www.cosmos.esa.int/web/gaia/dpac/consortium). Funding for the DPAC has been provided by national institutions, in particular the institutions participating in the Gaia Multilateral Agreement. This research has made use of the SIMBAD data base, operated at CDS, Strasbourg, France.

DM, VDO, RG, SD acknowledge support from the 'Progetti Premiali' funding scheme of the Italian Ministry of Education, University, and Research and from the ASI-INAF agreement no. 2018-16-HH.0. SM is supported by a research fellowship from Jesus College, University of Cambridge. MB acknowledges funding by the UK Science and Technology Facilities Council (STFC) grant no. ST/M001229/1. TH acknowledges support from the European Research Council under the Horizon 2020 Framework Programme via the ERC Advanced Grant Origins 832428.

\section{DATA AVAILABILITY}

The data underlying this article will be shared on reasonable request to the corresponding author. 


\section{REFERENCES}

Allard F., Guillot T., Ludwig H.-G., Hauschildt P. H., Schweitzer A., Alexander D. R., Ferguson J. W., 2003, in Martín E., ed., Proc. IAU Symp. 211, Brown Dwarfs. Astron. Soc. Pac., San Francisco, p. 325

ALMA Partnership et al., 2015, ApJ, 808, L3

Apai D. et al., 2008, ApJ, 672, 1196

Ardila D. R. et al., 2004, ApJ, 617, L147

Baranne A. et al., 1996, A\&AS, 119, 373

Beichman C. A., Rieke M., Eisenstein D., Greene T. P., Krist J., McCarthy D., Meyer M., Stansberry J., 2012, Proc. SPIE Conf. Ser. Vol. 8442, Space Telescopes and Instrumentation 2012: Optical, Infrared, and Millimeter Wave. SPIE, Bellingham, p. $84422 \mathrm{~N}$

Bell C. P. M., Mamajek E. E., Naylor T., 2015, MNRAS, 454, 593

Beuzit J. L. et al., 2019, A\&A, 631, A155

Biller B. A. et al., 2013, ApJ, 777, 160

Blanco-Cuaresma S., 2019, MNRAS, 486, 2075

Blanco-Cuaresma S., Soubiran C., Heiter U., Jofré P., 2014, A\&A, 569, A111

Boccaletti A. et al., 2015, Nature, 526, 230

Boccaletti A. et al., 2019, A\&A, 625, A21

Bonavita M., 2020, Exo-DMC: Exoplanet Detection Map Calculator, ASCL, record ascl:2010.008

Bonavita M., Chauvin G., Desidera S., Gratton R., Janson M., Beuzit J. L., Kasper M., Mordasini C., 2012, A\&A, 537, A67

Bonavita M., de Mooij E. J. W., Jayawardhana R., 2013, PASP, 125 , 849

Bonnefoy M. et al., 2017, A\&A, 597, L7

Booth M. et al., 2016, MNRAS, 460, L10

Bouchy F., Sophie Team, 2006, in Arnold L., Bouchy F., Moutou C., eds, Tenth Anniversary of 51 Peg-b: Status of and Prospects for Hot Jupiter Studies. Frontier Group, Paris, p. 319

Bouchy F. et al., 2009, A\&A, 505, 853

Bouchy F., Díaz R. F., Hébrard G., Arnold L., Boisse I., Delfosse X., Perruchot S., Santerne A., 2013, A\&A, 549, A49

Burggraaff O. et al., 2018, A\&A, 617, A32

Castelli F., Kurucz R. L., 2003, in Piskunov N., Weiss W. W., Gray D. F., eds, Proc. IAU Symp. 210, Modelling of Stellar Atmospheres. Astron. Soc. Pac., San Francisco, p. A20

Chauvin G. et al., 2017, in Reylé C., Di Matteo P., Herpin F., Lagadec E., Lançon A., Meliani Z., Royer F., eds, Proc. Ann. Meeting French Soc. Astron. Astrophys. (SF2A-2017). French Society of Astronomy and Astrophysics, Paris, p. 331

Chen C. H. et al., 2005, ApJ, 634, 1372

Chen C. H., Pecaut M., Mamajek E. E., Su K. Y. L., Bitner M., 2012, ApJ, 756, 133

Claudi R. U. et al., 2008, Proc. SPIE Conf. Ser. Vol. 7014, Ground-based and Airborne Instrumentation for Astronomy II. SPIE, Bellingham, p. 70143E

da Silva L. et al., 2006, A\&A, 458, 609

Delorme P. et al., 2017, in Reylé C., Di Matteo P., Herpin F., Lagadec E., Lançon A., Meliani Z., Royer F., eds, Proc. Ann. Meeting French Soc. Astron. Astrophys. (SF2A-2017). French Society of Astronomy and Astrophysics, Paris, p. 347

Dent W. R. F. et al., 2014, Science, 343, 1490

Desidera S. et al., 2015, A\&A, 573, A126

Dohlen K. et al., 2008, Proc. SPIE Conf. Ser. Vol. 7014, Ground-based and Airborne Instrumentation for Astronomy II. SPIE, Bellingham, p. 70143L

D’Orazi V. et al., 2020, A\&A, 633, A38

Engler N. et al., 2019, A\&A, 622, A192

Ertel S., Wolf S., Metchev S., Schneider G., Carpenter J. M., Meyer M. R., Hillenbrand L. A., Silverstone M. D., 2011, A\&A, 533, A132

Fedele D. et al., 2018, A\&A, 610, A24

Feldt M. et al., 2017, A\&A, 601, A7

Gagné J. et al., 2018, ApJ, 856, 23

Gaia Collaboration, 2016, A\&A, 595, A1

Gaia Collaboration, 2018, A\&A, 616, A1

Galicher R. et al., 2018, A\&A, 615, A92
Gladman B., 1993, Icarus, 106, 247

Golimowski D. A. et al., 2011, AJ, 142, 30

Haffert S. Y., Bohn A. J., de Boer J., Snellen I. A. G., Brinchmann J., Girard J. H., Keller C. U., Bacon R., 2019, Nat. Astron., 3, 749

Harlan E. A., Taylor D. C., 1970, AJ, 75, 507

Herbst W., Bailer-Jones C. A. L., Mundt R., Meisenheimer K., Wackermann R., 2002, A\&A, 396, 513

Hogg D. W., Myers A. D., Bovy J., 2010, ApJ, 725, 2166

Isella A. et al., 2018, ApJ, 869, L49

Janson M. et al., 2013, ApJ, 773, 73

Kalas P., Graham J. R., Clampin M., 2005, Nature, 435, 1067

Kaufer A., Stahl O., Tubbesing S., Nørregaard P., Avila G., Francois P. Pasquini L., Pizzella A., 1999, The Messenger, 95, 8

Kennedy G. M., Wyatt M. C., 2014, MNRAS, 444, 3164

Keppler M. et al., 2018, A\&A, 617, A44

Kervella P., Arenou F., Mignard F., Thévenin F., 2019, A\&A, 623, A72

Lagrange A. M. et al., 2009, A\&A, 493, L21

Lamm M. H., Bailer-Jones C. A. L., Mundt R., Herbst W., Scholz A., 2004, A\&A, 417, 557

Lannier J. et al., 2017, A\&A, 603, A54

Lazzoni C. et al., 2018, A\&A, 611, A43

Lo Curto G. et al., 2015, The Messenger, 162, 9

Lombart M. et al., 2020, A\&A, 639, A54

Lovis C. et al., 2006, Proc. SPIE Conf. Ser. Vol. 6269, Ground-based and Airborne Instrumentation for Astronomy. SPIE, Bellingham, p. 62690P

MacGregor M. A. et al., 2019, ApJ, 877, L32

Macintosh B. et al., 2006, Proc. SPIE Conf. Ser. Vol. 6272, Advances in Adaptive Optics II. SPIE, Bellingham, p. 62720L

Mamajek E. E., Hillenbrand L. A., 2008, ApJ, 687, 1264

Marino S. et al., 2018, MNRAS, 479, 5423

Marino S., Yelverton B., Booth M., Faramaz V., Kennedy G. M., Matrà L., Wyatt M. C., 2019, MNRAS, 484, 1257

Marino S. et al., 2020, MNRAS, 498, 1319

Marois C., Lafrenière D., Doyon R., Macintosh B., Nadeau D., 2006, ApJ, 641,556

Marois C., Macintosh B., Barman T., Zuckerman B., Song I., Patience J., Lafrenière D., Doyon R., 2008, Science, 322, 1348

Marois C., Zuckerman B., Konopacky Q. M., Macintosh B., Barman T., 2010, Nature, 468, 1080

Marois C., Correia C., Galicher R., Ingraham P., Macintosh B., Currie T., De Rosa R., 2014, Proc. SPIE Conf. Ser. Vol. 9148, Adaptive Optics Systems IV. SPIE, Bellingham, p. $91480 \mathrm{U}$

Marshall J. P. et al., 2014, A\&A, 565, A15

Marzari F., 2014, MNRAS, 442, 1110

Matthews E. et al., 2018, MNRAS, 480, 2757

Mawet D. et al., 2014, ApJ, 792, 97

Meibom S., Mathieu R. D., Stassun K. G., 2009, ApJ, 695, 679

Meibom S., Mathieu R. D., Stassun K. G., Liebesny P., Saar S. H., 2011, ApJ, 733,115

Mesa D. et al., 2015, A\&A, 576, A121

Mesa D. et al., 2019a, A\&A, 632, A25

Mesa D. et al., 2019b, MNRAS, 488, 37

Meshkat T., Bailey V. P., Su K. Y. L., Kenworthy M. A., Mamajek E. E., Hinz P. M., Smith P. S., 2015, ApJ, 800, 5

Meunier N., Lagrange A. M., De Bondt K., 2012, A\&A, 545, A87

Milli J., Mouillet D., Lagrange A. M., Boccaletti A., Mawet D., Chauvin G., Bonnefoy M., 2012, A\&A, 545, A111

Morales F. Y., Rieke G. H., Werner M. W., Bryden G., Stapelfeldt K. R., Su K. Y. L., 2011, ApJ, 730, L29

Morrison S., Malhotra R., 2015, ApJ, 799, 41

Müller A. et al., 2018, A\&A, 617, L2

Nederlander A. et al. , 2021, preprint (arXiv: 2101.08849)

Nesvold E. R., Kuchner M. J., 2015, ApJ, 798, 83

Nielsen E. L. et al., 2019, AJ, 158, 13

Nordström B. et al., 2004, A\&A, 418, 989

Noyes R. W., Hartmann L. W., Baliunas S. L., Duncan D. K., Vaughan A. H., 1984, ApJ, 279, 763 
Pavlov A., Feldt M., Henning T., 2008, in Argyle R. W., Bunclark P. S., Lewis J. R., eds, ASP Conf. Ser. Vol. 394, Astronomical Data Analysis Software and Systems XVII. Astron. Soc. Pac., San Francisco, p. 581

Pearce T. D., Wyatt M. C., 2014, MNRAS, 443, 2541

Pearce T. D., Wyatt M. C., 2015, MNRAS, 453, 3329

Pecaut M. J., Mamajek E. E., 2013, ApJS, 208, 9

Perrot C. et al., 2016, A\&A, 590, L7

Perruchot S. et al., 2008, Proc. SPIE Conf. Ser. Vol. 7014, Ground-based and Airborne Instrumentation for Astronomy II. SPIE, Bellingham, p. 70140J

Perruchot S. et al., 2011, Proc. SPIE Conf. Ser. Vol. 8151, Techniques and Instrumentation for Detection of Exoplanets V. SPIE, Bellingham, p. 815115

Pinilla P. et al., 2018, ApJ, 859, 32

Plavchan P., Werner M. W., Chen C. H., Stapelfeldt K. R., Su K. Y. L., Stauffer J. R., Song I., 2009, ApJ, 698, 1068

Press W. H., Teukolsky S. A., Vetterling W. T., Flannery B. P., 2002, Numerical Recipes in $\mathrm{C}++$ : The Art of Scientific Computing

Quillen A. C., Faber P., 2006, MNRAS, 373, 1245

Rameau J. et al., 2013, ApJ, 779, L26

Rameau J. et al., 2016, ApJ, 822, L29

Ramírez I. et al., 2014, A\&A, 572, A48

Read M. J., Wyatt M. C., Marino S., Kennedy G. M., 2018, MNRAS, 475, 4953

Rebull L. M. et al., 2016, AJ, 152, 113

Ricci L., Carpenter J. M., Fu B., Hughes A. M., Corder S., Isella A., 2015, ApJ, 798, 124

Ricker G. R. et al., 2015, J. Astron. Telesc. Instrum. Syst., 1, 014003

Roberts D. H., Lehar J., Dreher J. W., 1987, AJ, 93, 968

Scargle J. D., 1982, ApJ, 263, 835

Sefilian A. A., Rafikov R. R., Wyatt M. C., 2020, preprint (arXiv:2010.15617)

Shannon A., Bonsor A., Kral Q., Matthews E., 2016, MNRAS, 462, L116

Silverstone M. D., 2000, PhD thesis, Univ. California, Los Angeles

Sneden C. A., 1973, PhD thesis, Univ. Texas at Austin

Song I., Zuckerman B., Bessell M. S., 2004, ApJ, 614, L125

Soummer R., Pueyo L., Larkin J., 2012, ApJ, 755, L28

Sousa S. G., Santos N. C., Adibekyan V., Delgado-Mena E., Israelian G., 2015, A\&A, 577, A67

Strassmeier K., Washuettl A., Granzer T., Scheck M., Weber M., 2000, A\&AS, 142, 275

Su K. Y. L., Morrison S., Malhotra R., Smith P. S., Balog Z., Rieke G. H., 2015, ApJ, 799, 146

Torres C. A. O., Quast G. R., da Silva L., de La Reza R., Melo C. H. F., Sterzik M., 2006, A\&A, 460, 695

Valenti J. A., Fischer D. A., 2005, ApJS, 159, 141

van Boekel R. et al., 2017, ApJ, 837, 132

van Leeuwen F., 2007, A\&A, 474, 653

Vigan A., Moutou C., Langlois M., Allard F., Boccaletti A., Carbillet M., Mouillet D., Smith I., 2010, MNRAS, 407, 71

Vigan A. et al., 2017, A\&A, 603, A3

Vigan A. et al., 2020, preprint (arXiv:2007.06573)

Wichmann R., Schmitt J. H. M. M., Hubrig S., 2003, A\&A, 399, 983

Williams J. P., Najita J., Liu M. C., Bottinelli S., Carpenter J. M., Hillenbrand L. A., Meyer M. R., Soderblom D. R., 2004, ApJ, 604, 414

Wisdom J., 1980, AJ, 85, 1122

Wright J. T., Marcy G. W., Butler R. P., Vogt S. S., 2004, ApJS, 152, 261

Wyatt M. C., 2006, ApJ, 639, 1153

Zheng X., Lin D. N. C., Kouwenhoven M. B. N., Mao S., Zhang X., 2017, ApJ, 849, 98
${ }^{1}$ INAF-Osservatorio Astronomico di Padova, Vicolo dell'Osservatorio 5, Padova, I-35122, Italy

${ }^{2}$ Max Planck Institute for Astronomy, Königstuhl 17, D-69117 Heidelberg, Germany

${ }^{3}$ Institute of Astronomy, University of Cambridge, Madingley Road, Cambridge CB3 OHA, UK

${ }^{4}$ SUPA, Institute for Astronomy, University of Edinburgh, Blackford Hill, Edinburgh EH9 3HJ, UK

${ }^{5}$ Centre for Exoplanet Science, University of Edinburgh, Edinburgh EH9 $3 \mathrm{HJ}$, $U K$

${ }^{6}$ Dipartimento di Fisica a Astronomia 'G. Galilei', Universit'a di Padova, Via Marzolo, 8, I-35121 Padova, Italy

${ }^{7}$ Center for Space and Habitability, University of Bern, Gesellschaftsstrasse 6. CH-3012 Bern, Switzerland

${ }^{8}$ Universidad de Santiago de Chile, Av. Libertador Bernardo O'Higgins 3363, Estacion Central, Santiago, Chile

${ }^{9}$ School of Physics and Astronomy, Monash University, Clayton Campus, VIC 3800, Australia

${ }^{10}$ ETH Zurich, Institute for Particle Physics and Astrophysics, WolfgangPauli-Strasse 27, CH-8093 Zurich, Switzerland

${ }^{11}$ Department of Astronomy, Stockholm University, 10691, Stockholm, Sweden

${ }^{12}$ LESIA, Observatoire de Paris, Université PSL, CNRS, Sorbonne Université, Univ. Paris Diderot, Sorbonne Paris Cité, 5 place Jules Janssen, F-92195 Meudon, France

${ }^{13}$ Univ. Lyon, Univ. Lyon 1, ENS de Lyon, CNRS, CRAL UMR 5574, F-69230 Saint-Genis-Laval, France

${ }^{14}$ Aix Marseille Univ., CNRS, CNES, LAM, Marseille, France

${ }^{15}$ INAF-Catania Astrophysical Observatory, Via S. Sofia, 78, I-95123 Catania, Italy

${ }^{16}$ Univ. Grenoble Alpes, CNRS, IPAG, F-38000 Grenoble, France

${ }^{17}$ Konkoly Observatory, Research Centre for Astronomy and Earth Sciences, Konkoly-Thege Miklós út 15-17, H-1121 Budapest, Hungary

${ }^{18}$ Instituto de Física y Astronomía, Facultad de Ciencias, Universidad de Valparaíso,, Av. Gran Bretaña 1111, Playa Ancha, Valparaíso, Chile

${ }^{19}$ Núcleo Milenio Formación Planetaria - NPF, Universidad de Valparaíso, Av. Gran Bretana 1111, Playa Ancha, Valparaíso, Chile

${ }^{20}$ Geneva Observatory, University of Geneva, Chemin des Maillettes 51, $\mathrm{CH}$ 1290 Sauverny, Switzerland

${ }^{21}$ European Space Agency (ESA), ESA Office, Space Telescope Science Institute, 3700 San Martin Drive, Baltimore, MD 21218, USA

${ }^{22}$ European Southern Observatory, Alonso de Còrdova 3107, Vitacura, Casilla 19001, Santiago, Chile

${ }^{23}$ Nucleo de Astronomia, Facultad de Ingenieria y Ciencias, Universidad Diego Portales, Av. Ejercito 441, Santiago, Chile

${ }^{24}$ Escuela de Ingenieria Industrial, Facultad de Ingenieria y Ciencias, Universidad Diego Portales, Av. Ejercito 441, Santiago, Chile

${ }^{25}$ Departamento de Astronomía, Universidad de Chile, Casilla 36-D, Santiago

${ }^{26}$ INAF - Osservatorio Astronomico di Capodimonte, Via Salita Moiariello 16, I-80131 Napoli, Italy

${ }^{27}$ STAR Institute, Université de Liege, Allee du Six Août 19c, B-4000 Liege, Belgium

${ }^{28}$ University of Michigan, Astronomy Department, 1085 S. University Ave., Ann Arbor, MI, 48109-1107, USA

This paper has been typeset from a $\mathrm{T}_{\mathrm{E}} \mathrm{X} / \mathrm{L} \mathrm{T} \mathrm{E} \mathrm{X}$ file prepared by the author. 